\title{
, Dual Pairs of Generalized Lyapunov Inequalities and Balanced Truncation of Stochastic Linear Systems
}

\author{
Peter Benner, Tobias Damm, and Yolanda Rocio Rodriguez Cruz
}

\begin{abstract}
4 Abstract-We consider two approaches to balanced truncation 5 of stochastic linear systems, which follow from different general6 izations of the reachability Gramian of deterministic systems. Both 7 preserve mean-square asymptotic stability, but only the second 8 leads to a stochastic $\boldsymbol{H}^{\infty}$-type bound for the approximation error 9 of the truncated system.
\end{abstract}

10 Index Terms-Asymptotic mean square stability, balanced trun11 cation, generalized Lyapunov equation, model order reduction, 12 stochastic linear system.

\section{INTRODUCTION}

"O PTIMIZATION and (feedback) control of dynamical systems is often computationally infeasible for high dimen17 sional plant models. Therefore, one tries to reduce the order of 18 the system, so that the input-output mapping is still computable 19 with sufficient accuracy, but at considerably smaller cost than 20 for the original system [1]-[5]. To guarantee the desired accu21 racy, computable error bounds are required. Moreover, system 22 properties which are relevant in the context of control system 23 design like asymptotic stability need to be preserved. It has 24 long been known that for linear time-invariant (LTI) systems the 25 method of balanced truncation preserves asymptotic stability 26 and provides an error bound for the $L^{2}$-induced input-output 27 norm, i.e., the $H^{\infty}$-norm of the associated transfer function; 28 see [6], [7]. When considering model order reduction of more 29 general system classes, it is natural to try to extend this ap30 proach. This has been worked out for descriptor systems in 31 [8], for time-varying systems in [9]-[11], for bilinear systems 32 in [12]-[14] and general nonlinear systems, e.g., in [15]. Yet 33 another generalization of LTI systems is obtained considering 34 dynamics driven by noise processes. This leads to the class of 35 stochastic systems, which have been considered in a system 36 theoretic context, e.g., in [16]-[18]. Quite recently, balanced 37 truncation has also been described for linear stochastic systems 38 of Itô type in [14], [19], and [20]. Already the formulation of 39 the method leads to two different variants that are equivalent 40 in the deterministic case, but not so for stochastic systems. It 41 is natural to ask which of the above-mentioned properties of

Manuscript received December 18, 2015; accepted April 25, 2016. Recommended by Associate Editor J. Imura.

P. Benner is with Max Planck Institute for Dynamics of Complex Technical Systems, 39106 Magdeburg, Germany (e-mail: benner@mpi-magdeburg. mpg.de).

T. Damm and Y. R. Rodriguez Cruz are with the Department of Mathematics, University of Kaiserslautern, 67663 Kaiserslautern, Germany (e-mail: damm@ mathematik.uni-kl.de; rodrigue@ mathematik.uni-kl.de).

Color versions of one or more of the figures in this paper are available online at http://ieeexplore.ieee.org.

Digital Object Identifier 10.1109/TAC.2016.2572881 balanced truncation also hold for these variants. The aim of this 42 paper is to answer this question.

Let us recapitulate balanced truncation for linear control 44 systems of the form

$$
\dot{x}=A x+B u \quad y=C x \quad \sigma(A) \subset \mathbb{C}_{-} .
$$

Here $A \in \mathbb{R}^{n \times n}, B \in \mathbb{R}^{n \times m}, C \in \mathbb{R}^{p \times n}$, and $x(t) \in \mathbb{R}^{n}, y(t) \in 46$ $\mathbb{R}^{p}$ and $u(t) \in \mathbb{R}^{m}$ are the state, output, and input of the system, 47 respectively. Moreover $\sigma(A)$ denotes the spectrum of $A$ and $\mathbb{C}_{-} 48$ the open left half complex plane. Let

$$
\mathcal{L}_{A}: X \mapsto A^{T} X+X A
$$

denote the Lyapunov operator and

$$
\mathcal{L}_{A}^{*}: X \mapsto A X+X A^{T}
$$

its adjoint with respect to the Frobenius inner product $\langle Z, Y\rangle=51$ $\operatorname{trace}\left(Y^{T} Z\right)$. Then $\sigma(A) \subset \mathbb{C}_{-}$if and only if there exists a posi- 52 tive definite solution $X$ of the Lyapunov inequality $\mathcal{L}_{A}(X)<0,53$ by Lyapunov's classical stability theorem, see, e.g., [21]. $\quad 54$

Balanced truncation means truncating a balanced realization. 55 This realization is obtained by a state space transformation 56 computed from the Gramians $P$ and $Q$, which solve the dual 57 pair of Lyapunov equations

$$
\begin{aligned}
& \mathcal{L}_{A}(Q)=A^{T} Q+Q A=-C^{T} C \\
& \mathcal{L}_{A}^{*}(P)=A P+P A^{T}=-B B^{T}
\end{aligned}
$$

or more generally the inequalities

$$
\mathcal{L}_{A}(Q) \leq-C^{T} C \quad \mathcal{L}_{A}^{*}(P) \leq-B B^{T} .
$$

These (in)equalities are essential in the characterization of 60 stability, controllability and observability of system (1). If 61 $\operatorname{det} P \neq 0$, the inequalities (3) can be written as

$$
\begin{aligned}
\mathcal{L}_{A}(Q) & \leq-C^{T} C \\
\mathcal{L}_{A}\left(P^{-1}\right) & =P^{-1} A+A^{T} P^{-1} \leq-P^{-1} B B^{T} P^{-1} .
\end{aligned}
$$

In the present paper we discuss extensions of (3) and (4) for 63 stochastic linear systems.

As indicated above, the equivalent formulations (3) and (4) 65 lead to different generalizations, if we consider Itô-type sto- 66 chastic systems of the form

$$
d x=A x d t+N x d w+B u d t, \quad y=C x
$$

where $A, B, C$ are as in (1) and $N \in \mathbb{R}^{n \times n}$. System (5) is 68 asymptotically mean-square stable (e.g., [18], [22], [23]), if and 69 
70 only if there exists a positive definite solution $X$ of the gener71 alized Lyapunov inequality

$$
\left(\mathcal{L}_{A}+\Pi_{N}\right)(X)=A^{T} X+X A+N^{T} X N<0 .
$$

72 Here $\Pi_{N}: X \mapsto N^{T} X N$ and $\Pi_{N}^{*}: X \mapsto N X N^{T}$. This sta73 bility criterion indicates that in the stochastic context, the 74 generalized Lyapunov operator $\mathcal{L}_{A}+\Pi_{N}$ takes over the role 75 of $\mathcal{L}_{A}$. Substituting $\mathcal{L}_{A}$ by $\mathcal{L}_{A}+\Pi_{N}$ in (3) and (4), we obtain 76 two different dual pairs of generalized Lyapunov inequalities. 77 We call them type I

$$
\begin{aligned}
\left(\mathcal{L}_{A}+\Pi_{N}\right)(Q) & =A^{T} Q+Q A+N^{T} Q N \leq-C^{T} C \\
\left(\mathcal{L}_{A}+\Pi_{N}\right)^{*}(P) & =A P+P A^{T}+N P N^{T} \leq-B B^{T}
\end{aligned}
$$

78 and type II

$$
\begin{aligned}
\left(\mathcal{L}_{A}+\Pi_{N}\right)(Q) & =A^{T} Q+Q A+N^{T} Q N \\
& \leq-C^{T} C \\
\left(\mathcal{L}_{A}+\Pi_{N}\right)\left(P^{-1}\right) & =A^{T} P^{-1}+P^{-1} A+N^{T} P^{-1} N \\
& \leq-P^{-1} B B^{T} P^{-1} .
\end{aligned}
$$

79 Note that (6) corresponds to (3) in the sense that $\mathcal{L}_{A}^{*}(P)$ has 80 been replaced by $\left(\mathcal{L}_{A}+\Pi_{N}\right)^{*}(P)$, while (7) corresponds to 81 (4), where $\mathcal{L}_{A}\left(P^{-1}\right)$ has been replaced by $\left(\mathcal{L}_{A}+\Pi_{N}\right)\left(P^{-1}\right)$. 82 In general (if $N$ and $P$ do not commute), the inequalities (6b) 83 and (7b) are not equivalent. At first glance it is not clear which 84 generalization is more appropriate.

85 If the system is asymptotically mean-square stable, then 86 for both types there are solutions $Q, P>0$. By a suitable 87 state space-transformation, it is possible to balance the system 88 such that $Q=P=\Sigma>0$ is diagonal. Consequently, the usual 89 procedure of balanced truncation can be applied to reduce the 90 order of (5). For simplicity, let us refer to this as type I or type II 91 balanced truncation.

92 Under natural assumptions, this reduction preserves mean93 square asymptotic stability. For type I, this nontrivial fact has 94 been proven in [24]. Moreover, in [20], an $H^{2}$-error bound 95 has been provided. However, different from the deterministic 96 case, there is no $H^{\infty}$-type error bound in terms of the truncated 97 entries in $\Sigma$. This will be shown in Example I.3.

98 In contrast, for type II, an $H^{\infty}$-type error bound has been 99 obtained in [19]. In the present paper, as one of our main 100 contributions, we show in Theorem II.2 that type II balanced 101 truncation also preserves mean-square asymptotic stability. The 102 proof differs significantly from the one given for type I. Using 103 this result, we are able to give a more compact proof of the error 104 bound, Theorem II.4, which exploits the stochastic bounded 105 real lemma [17].

106 We illustrate our results by analytical and numerical exam107 ples in Section IV.

\section{II. TYPE I BALANCED TRUNCATION}

109 Consider a stochastic linear control system of Itô-type

$$
d x=A x d t+\sum_{j=1}^{k} N_{j} x d w_{j}+B u d t, \quad y=C x
$$

where $w_{j}=\left(w_{j}(t)\right)_{t \in \mathbb{R}_{+}}$are uncorrelated zero-mean real 110 Wiener processes on a probability space $(\Omega, \mathcal{F}, \mu)$ with respect 111 to an increasing family $\left(\mathcal{F}_{t}\right)_{t \in \mathbb{R}_{+}}$of $\sigma$-algebras $\mathcal{F}_{t} \subset \mathcal{F}$ (e.g., 112 [25], [26]).

To simplify the notation, we only consider the case $k=1114$ and set $w=w_{1}, N=N_{1}$. But all results can immediately be 115 generalized for $k>1$.

Let $L_{w}^{2}\left(\mathbb{R}_{+}, \mathbb{R}^{q}\right)$ denote the corresponding space of nonan- 117 ticipating stochastic processes $v$ with values in $\mathbb{R}^{q}$ and norm 118

$$
\|v(\cdot)\|_{L_{w}^{2}}^{2}:=\mathcal{E}\left(\int_{0}^{\infty}\|v(t)\|^{2} d t\right)<\infty
$$

where $\mathcal{E}$ denotes expectation.

Let the homogeneous equation $d x=A x d t+N x d w$ be 120 asymptotically mean-square-stable, i.e., $\mathcal{E}\left(\|x(t)\|^{2}\right) \stackrel{t \rightarrow \infty}{\longrightarrow} 0$, for 121 all solutions $x$.

Then, by Theorem A.1, the equations

$$
\begin{aligned}
& A^{T} Q+Q A+N^{T} Q N=-C^{T} C \\
& A P+P A^{T}+N P N^{T}=-B B^{T}
\end{aligned}
$$

have unique solutions $Q \geq 0$ and $P \geq 0$. If the system is 124 observable and reachable (see Theorem A.8), then $Q$ and $P$ are 125 nonsingular, and thus positive definite.

A similarity transformation

$$
(A, N, B, C) \mapsto\left(S^{-1} A S, S^{-1} N S, S^{-1} B, C S\right)
$$

of the system implies the contragradient transformation as

$$
(Q, P) \mapsto\left(S^{T} Q S, S^{-1} P S^{-T}\right) .
$$

Choosing, e.g., $S=L V \Sigma^{-1 / 2}$, with Cholesky factorizations 129 $L L^{T}=P, R^{T} R=Q$ and a singular value decomposition 130 $R L=U \Sigma V^{T}$, we obtain $S^{-1}=\Sigma^{-1 / 2} U^{T} R$ and

$$
S^{T} Q S=S^{-1} P S^{-T}=\Sigma=\operatorname{diag}\left(\sigma_{1}, \ldots, \sigma_{n}\right) .
$$

After suitable partitioning

$$
\Sigma=\left[\begin{array}{cc}
\Sigma_{1} & 0 \\
0 & \Sigma_{2}
\end{array}\right] \quad S=\left[\begin{array}{ll}
S_{1} & S_{2}
\end{array}\right] \quad S^{-1}=\left[\begin{array}{l}
T_{1} \\
T_{2}
\end{array}\right]
$$

a truncated system is given in the form

$$
\left(A_{11}, N_{11}, B_{1}, C_{1}\right)=\left(T_{1} A S_{1}, T_{1} N S_{1}, T_{1} B, C S_{1}\right) .
$$

The following result has been proven in [24].

Theorem I.1: Let $A, N \in \mathbb{R}^{n \times n}$ satisfy

$$
\sigma(I \otimes A+A \otimes I+N \otimes N) \subset \mathbb{C}_{-} .
$$

For a block-diagonal matrix $\Sigma=\operatorname{diag}\left(\Sigma_{1}, \Sigma_{2}\right)>0$ with 136 $\sigma\left(\Sigma_{1}\right) \cap \sigma\left(\Sigma_{2}\right)=\emptyset$, assume that

$$
A^{T} \Sigma+\Sigma A+N^{T} \Sigma N \leq 0 \text { and } A \Sigma+\Sigma A^{T}+N \Sigma N^{T} \leq 0 .
$$

Then, with the usual partitioning of $A$ and $N$, we have 
139 Its implication for mean-square stability of the truncated system 140 is immediate.

141 Corollary I.2: Consider an asymptotically mean square sta142 ble stochastic linear system

$$
d x=A x d t+N x d w .
$$

143 Assume that a matrix $\Sigma=\operatorname{diag}\left(\Sigma_{1}, \Sigma_{2}\right)$ is given as in 144 Theorem I.1 and $A$ and $N$ are partitioned accordingly. Then the 145 truncated system

$$
d x_{r}=A_{11} x_{r} d t+N_{11} x_{r} d w
$$

146 is also asymptotically mean square stable.

147 If the diagonal entries of $\Sigma_{2}$ are small, it is expected that the 148 truncation error is small. In fact this is supported by an $\mathrm{H}^{2}$-error 149 bound obtained in [20]. Additionally, however, from the de150 terministic situation (see [2], [6]), one would also hope for an $151 H^{\infty}$-type error bound of the form

$$
\left\|y-y_{r}\right\|_{L_{w}^{2}\left(\mathbb{R}_{+}, \mathbb{R}^{p}\right)} \stackrel{?}{\leq} \alpha\left(\operatorname{trace} \Sigma_{2}\right)\|u\|_{L_{w}^{2}\left(\mathbb{R}_{+}, \mathbb{R}^{m}\right)}
$$

152 with some real number $\alpha>0$. The following example shows 153 that no such general $\alpha$ exists.

154 Example I.3: Let $A=-\left[\begin{array}{cc}1 & 0 \\ 0 & a^{2}\end{array}\right]$ with $a>1, \quad N=$ $155\left[\begin{array}{ll}0 & 0 \\ 1 & 0\end{array}\right], B=\left[\begin{array}{l}1 \\ 0\end{array}\right], C=\left[\begin{array}{ll}0 & 1\end{array}\right]$.

156 Solving (6) with equality, we get $P=\left[\begin{array}{cc}\frac{1}{2} & 0 \\ 0 & \frac{1}{4 a^{2}}\end{array}\right], Q=$ $157\left[\begin{array}{cc}\frac{1}{4 a^{2}} & 0 \\ 0 & \frac{1}{2 a^{2}}\end{array}\right]$ with $\sigma(P Q)=\left\{1 / 8 a^{2}, 1 / 8 a^{4}\right\}$ so that $\Sigma=$ $158 \operatorname{diag}\left(\sigma_{1}, \sigma_{2}\right)$, where $\sigma_{1}=1 / \sqrt{8} a$ and $\sigma_{2}=1 / \sqrt{8} a^{2}$. The sys159 tem is balanced by the transformation $S=\left[\begin{array}{cc}2 a^{2} & 0 \\ 0 & 1 / 2\end{array}\right]^{1 / 4}$. 160 Then $C S=\left(1 / 2^{1 / 4}\right)\left[\begin{array}{ll}0 & 1\end{array}\right]$ so that $C_{r}=0$ for the trun161 cated system of order 1 . Thus, the output of the reduced system 162 is $y_{r} \equiv 0$, and the truncation error $\left\|\mathbb{L}-\mathbb{L}_{r}\right\|$ is equal to the 163 stochastic $H^{\infty}$-norm (see [17]) of the original system

$$
\|\mathbb{L}\|=\sup _{x(0)=0,\|u\|_{L_{w}^{2}=1}}\|y\|_{L_{w}^{2}} \text {. }
$$

164 We show now that this norm is equal to $1 / \sqrt{2} a=2 a \sigma_{2}$. 165 Thus, depending on $a$, the ratio of the truncation error and 166 trace $\Sigma_{2}=\sigma_{2}$ can be arbitrarily large.

167 According to the stochastic bounded real lemma, 168 Theorem A.5, $\|\mathbb{L}\|$ is the infimum over all $\gamma$ so that the Riccati 169 inequality

$$
\begin{aligned}
0 & <A^{T} X+X A+N^{T} X N-C^{T} C-\frac{1}{\gamma^{2}} X B B^{T} X \\
& =\left[\begin{array}{cc}
-2 x_{1}+x_{3}-\frac{1}{\gamma^{2}} x_{1}^{2} & -\left(a^{2}+1\right) x_{2}-\frac{1}{\gamma^{2}} x_{1} x_{2} \\
-\left(a^{2}+1\right) x_{2}-\frac{1}{\gamma^{2}} x_{1} x_{2} & -2 a^{2} x_{3}-\frac{1}{\gamma^{2}} x_{2}^{2}-1
\end{array}\right]
\end{aligned}
$$

170 possesses a solution $X=\left[\begin{array}{ll}x_{1} & x_{2} \\ x_{2} & x_{3}\end{array}\right]<0$.
If a given matrix $X$ satisfies this condition, then so does the 171 same matrix with $x_{2}$ replaced by 0 . Hence we can assume that 172 $x_{2}=0$, and end up with the two conditions $x_{3}<-\left(1 / 2 a^{2}\right) 173$ and (after multiplying the upper left entry with $-\gamma^{2}$ )

$$
\begin{aligned}
0 & >x_{1}^{2}+2 \gamma^{2} x_{1}-\gamma^{2} x_{3}=\left(x_{1}+\gamma^{2}\right)^{2}-\gamma^{2}\left(\gamma^{2}+x_{3}\right) \\
& >\left(x_{1}+\gamma^{2}\right)^{2}-\gamma^{2}\left(\gamma^{2}-\frac{1}{2 a^{2}}\right) .
\end{aligned}
$$

Thus necessarily $\gamma^{2}>1 / 2 a^{2}$, i.e., $\gamma>1 / \sqrt{2} a$. This already 175 proves that $\|\mathbb{L}\| \geq 1 / \sqrt{2} a=2 a \sigma_{2}$, which suffices to disprove 176 the existence of a general bound $\alpha$ in (9). Taking infima, it is 177 AQ1 easy to show that indeed $\|\mathbb{L}\|=1 / \sqrt{2} a$.

\section{TYPE II BALANCED TRUNCATION}

We now consider the inequalities (7).

Lemma II.1: Assume that $d x=A x d t+N x d w$ is asymptot- 181 ically mean-square-stable. Then inequality (7b) is solvable with 182 $P>0$.

Proof: By Theorem A.1, for a given $Y<0$, there exists a 184 $\tilde{P}>0$, so that $A^{T} \tilde{P}^{-1}+\tilde{P}^{-1} A+N^{T} \tilde{P}^{-1} N=Y$. Then $P=185$ $\varepsilon^{-1} \tilde{P}$, for sufficiently small $\varepsilon>0$, satisfies 186

$$
A^{T} P^{-1}+P^{-1} A+N^{T} P^{-1} N=\varepsilon Y<-\varepsilon^{2} \tilde{P}^{-1} B B^{T} \tilde{P}^{-1}
$$

so that ( $7 \mathrm{~b}$ ) holds even in the strict form.

It is easy to see that like in the previous section a state space 188 transformation

$$
(A, N, B, C) \mapsto\left(S^{-1} A S, S^{-1} N S, S^{-1} B, C S\right)
$$

leads to a contragradient transformation $Q \mapsto S^{T} Q S, P \mapsto 190$ $S^{-1} P S^{-T}$ of the solutions. That is, $Q$ and $P$ satisfy (7a) 191 and (7b), if and only if $S^{T} Q S$ and $S^{-1} P S^{-T}$ do so for the 192 transformed data. As before, we can assume the system to be 193 balanced with

$$
Q=P=\Sigma=\operatorname{diag}\left(\sigma_{1} I, \ldots, \sigma_{\nu} I\right)=\left[\begin{array}{ll}
\Sigma_{1} & \\
& \Sigma_{2}
\end{array}\right]
$$

where $\sigma_{1}>\cdots>\sigma_{\nu}>0$ and $\sigma\left(\Sigma_{1}\right)=\left\{\sigma_{1}, \ldots, \sigma_{r}\right\}, \sigma\left(\Sigma_{2}\right)=195$ $\left\{\sigma_{r+1}, \ldots, \sigma_{\nu}\right\}$. Hence, we will now assume (after balancing) 196 that a diagonal matrix $\Sigma$ as in (11) is given which satisfies

$$
\begin{gathered}
A^{T} \Sigma+\Sigma A+N^{T} \Sigma N \leq-C^{T} C \\
A^{T} \Sigma^{-1}+\Sigma^{-1} A+N^{T} \Sigma^{-1} N \leq-\Sigma^{-1} B B^{T} \Sigma^{-1} .
\end{gathered}
$$

Partitioning $A, N, B, C$ like $\Sigma$, we write the system as

$$
\begin{aligned}
d x_{1} & =\left(A_{11} x_{1}+A_{12} x_{2}+B_{1} u\right) d t+\left(N_{11} x_{1}+N_{12} x_{2}\right) d w \\
d x_{2} & =\left(A_{21} x_{1}+A_{22} x_{2}+B_{2} u\right) d t+\left(N_{21} x_{1}+N_{22} x_{2}\right) d w \\
y & =C_{1} x_{1}+C_{2} x_{2} .
\end{aligned}
$$

The reduced system obtained by truncation is

$$
d x_{r}=\left(A_{11} x_{r}+B_{1} u\right) d t+N_{11} x_{r} d w \quad y_{r}=C_{1} x_{r}
$$


200 The index $r$ is the number of different singular values $\sigma_{j}$ that 201 have been kept in the reduced system. In the following subsec202 tions, we consider matrices:

$$
A=\left[\begin{array}{ll}
A_{11} & A_{12} \\
A_{21} & A_{22}
\end{array}\right] \quad N=\left[\begin{array}{ll}
N_{11} & N_{12} \\
N_{21} & N_{22}
\end{array}\right]
$$

$203 \Sigma=\operatorname{diag}\left(\Sigma_{1}, \Sigma_{2}\right)$ as in (11), and equations of the form

$$
\begin{aligned}
A^{T} \Sigma+\Sigma A+N^{T} \Sigma N & =-\tilde{C}^{T} \tilde{C} \\
A^{T} \Sigma^{-1}+\Sigma^{-1} A+N^{T} \Sigma^{-1} N & =-\tilde{B} \tilde{B}^{T}
\end{aligned}
$$

204 with arbitrary right-hand sides $-\tilde{C}^{T} \tilde{C} \leq 0$ and $-\tilde{B} \tilde{B}^{T} \leq 0$.

\section{A. Preservation of Asymptotic Stability}

206 The following theorem is the main new result of this paper.

207 Theorem II.2: Let $A$ and $N$ be given such that

$$
\sigma(I \otimes A+A \otimes I+N \otimes N) \subset \mathbb{C}_{-} .
$$

208 Assume further that for a block-diagonal matrix $\Sigma=$ $209 \operatorname{diag}\left(\Sigma_{1}, \Sigma_{2}\right)>0$ with $\sigma\left(\Sigma_{1}\right) \cap \sigma\left(\Sigma_{2}\right)=\emptyset$, we have

$$
\begin{array}{r}
A^{T} \Sigma+\Sigma A+N^{T} \Sigma N \leq 0 \\
A^{T} \Sigma^{-1}+\Sigma^{-1} A+N^{T} \Sigma^{-1} N \leq 0 .
\end{array}
$$

210 Then, with the usual partitioning of $A$ and $N$, we have

$$
\sigma\left(I \otimes A_{11}+A_{11} \otimes I+N_{11} \otimes N_{11}\right) \subset \mathbb{C}_{-} .
$$

211 Again we have an immediate interpretation in terms of mean212 square stability of the truncated system.

213 Corollary II.3: Consider an asymptotically mean square 214 stable stochastic linear system

$$
d x=A x d t+N x d w .
$$

215 Assume that a matrix $\Sigma=\operatorname{diag}\left(\Sigma_{1}, \Sigma_{2}\right)$ is given as in 216 Theorem II.2 and $A$ and $N$ are partitioned accordingly. Then 217 the truncated system

$$
d x_{r}=A_{11} x_{r} d t+N_{11} x_{r} d w
$$

218 is also asymptotically mean square stable.

219 Proof of Theorem II.2: Note that the inequalities (15) are 220 equivalent to the equations (13) with appropriate right-hand 221 sides $-\tilde{C}^{T} \tilde{C}$ and $-\tilde{B} \tilde{B}^{T}$. In accordance with the partitioning 222 of $A, N$, and $\Sigma$, each matrix equation (13a) and (13b) consists 223 of three blocks.

224 By way of contradiction, we assume that (16) does not hold. 225 Then by Theorem A.3, there exist $V \geq 0, V \neq 0, \alpha \geq 0$ such that

$$
A_{11} V+V A_{11}^{T}+N_{11} V N_{11}^{T}=\alpha V .
$$

226 Taking the scalar product of the left upper block of (13a) with $227 V$, we obtain $0 \geq \alpha \operatorname{trace}\left(\Sigma_{1} V\right)$ whence $\alpha=0$ and $\tilde{C}_{1} V=0$, $228 N_{21} V=0$ by Corollary A.4. Hence

$$
\left(A_{11}^{T} \Sigma_{1}+\Sigma_{1} A_{11}+N_{11}^{T} \Sigma_{1} N_{11}\right) V=0 .
$$

229 Analogously, we have $\tilde{B}_{1}^{T} V=0$.
In particular, from $N_{21} V=0$, we get

$$
\left(\mathcal{L}_{A}^{*}+\Pi_{N}^{*}\right)\left(\left[\begin{array}{ll}
V & 0 \\
0 & 0
\end{array}\right]\right)=\left[\begin{array}{cc}
0 & V A_{21}^{T} \\
A_{21} V & 0
\end{array}\right] .
$$

We will show that $A_{21} V=0$, which implies

$$
0 \in \sigma(I \otimes A+A \otimes I+N \otimes N)
$$

in contradiction to (14), and thus finishes the proof.

We first show that $\operatorname{Im} V$ is invariant under $A_{11}$ and $N_{11}$. To 233 this end, let $V z=0$. Then by (17)

$$
0=z^{T}\left(A_{11} V+V A_{11}^{T}+N_{11} V N_{11}^{T}\right) z=z^{T} N_{11} V N_{11}^{T} z
$$

whence also $V N_{11}^{T} z=0$, i.e., $N_{11}^{T} z \in \operatorname{Ker} V$. From this, we have 235

$$
0=\left(A_{11} V+V A_{11}^{T}+N_{11} V N_{11}^{T}\right) z=V A_{11}^{T} z
$$

implying $A_{11}^{T} z \in \operatorname{Ker} V$. Thus, $A_{11}^{T} \operatorname{Ker} V \subset \operatorname{Ker} V$ and 236 $N_{11}^{T} \operatorname{Ker} V \subset \operatorname{Ker} V$.

237

Since $\operatorname{Ker} V=(\operatorname{Im} V)^{\top}$, it follows further that $\operatorname{Im} V$ is invari- 238 ant under $A_{11}$ and $N_{11}$.

Let $V=V_{1} V_{1}^{T}$, where $V_{1}$ has full column rank, i.e., 240 $\operatorname{det} V_{1}^{T} V_{1} \neq 0$. Then by the invariance, there exist square 241 matrices $X$ and $Y$, such that

$$
A_{11} V_{1}=V_{1} X \quad N_{11} V_{1}=V_{1} Y .
$$

It follows that

$$
\begin{aligned}
0 & =A_{11} V_{1} V_{1}^{T}+V_{1} V_{1}^{T} A_{11}^{T}+N_{11} V_{1} V_{1}^{T} N_{11}^{T} \\
& =V_{1}\left(X+X^{T}+Y Y^{T}\right) V_{1}^{T}
\end{aligned}
$$

whence $X+X^{T}+Y Y^{T}=0$. Moreover, from (18), we get

$$
\begin{aligned}
A_{11}^{T} \Sigma_{1} V_{1} & =-\Sigma_{1} A_{11} V_{1}-N_{11}^{T} \Sigma_{1} N_{11} V_{1} \\
& =-\Sigma_{1} V_{1} X-N_{11}^{T} \Sigma_{1} V_{1} Y .
\end{aligned}
$$

Using this substitution in the following computation, we obtain 245

$$
\begin{aligned}
0 \geq & V_{1}^{T} \Sigma_{1}^{2}\left(A_{11}^{T} \Sigma_{1}^{-1}+\Sigma_{1}^{-1} A_{11}+N_{11}^{T} \Sigma_{1}^{-1} N_{11}\right) \Sigma_{1}^{2} V_{1} \\
= & -V_{1}^{T} \Sigma_{1}^{3} V_{1} X-X^{T} V_{1}^{T} \Sigma_{1}^{3} V_{1} \\
& -V_{1}^{T} \Sigma_{1}^{2} N_{11}^{T} \Sigma_{1} V_{1} Y-Y^{T} V_{1}^{T} \Sigma_{1} N_{11} \Sigma_{1}^{2} V_{1} \\
& +V_{1}^{T} \Sigma_{1}^{2} N_{11}^{T} \Sigma_{1}^{-1} N_{11} \Sigma_{1}^{2} V_{1} .
\end{aligned}
$$

Taking the trace in (21), we have

$$
0=\operatorname{trace}\left[\begin{array}{c}
V_{1} Y \\
V_{1}
\end{array}\right]^{T} M\left[\begin{array}{c}
V_{1} Y \\
V_{1}
\end{array}\right]
$$

where

$$
M=\left[\begin{array}{cc}
\Sigma_{1}^{3} & -\Sigma_{1} N_{11} \Sigma_{1}^{2} \\
-\Sigma_{1}^{2} N_{11}^{T} \Sigma_{1} & \Sigma_{1}^{2} N_{11}^{T} \Sigma_{1}^{-1} N_{11} \Sigma_{1}^{2}
\end{array}\right]
$$

is positive semidefinite

$$
\left[\begin{array}{cc}
\Sigma_{1}^{3} & -\Sigma_{1} N_{11} \Sigma_{1}^{2} \\
-\Sigma_{1}^{2} N_{11}^{T} \Sigma_{1} & \Sigma_{1}^{2} N_{11}^{T} \Sigma_{1}^{-1} N_{11} \Sigma_{1}^{2}
\end{array}\right]\left[\begin{array}{c}
V_{1} Y \\
V_{1}
\end{array}\right]=0 .
$$


249 The first block row then implies $N_{11} \Sigma_{1}^{2} V_{1}=\Sigma_{1}^{2} V_{1} Y$. From 250 (21), using also (20) again, we thus have

$$
\begin{aligned}
0 & =\left(A_{11}^{T} \Sigma_{1}^{-1}+\Sigma_{1}^{-1} A_{11}+N_{11}^{T} \Sigma_{1}^{-1} N_{11}\right) \Sigma_{1}^{2} V_{1} \\
& =-\Sigma_{1} V_{1} X-N_{11}^{T} \Sigma_{1} V_{1} Y+\Sigma_{1}^{-1} A_{11} \Sigma_{1}^{2} V_{1}+N_{11}^{T} \Sigma_{1} V_{1} Y \\
& =-\Sigma_{1} V_{1} X+\Sigma_{1}^{-1} A_{11} \Sigma_{1}^{2} V_{1}
\end{aligned}
$$

251 i.e., $A_{11} \Sigma_{1}^{2} V_{1}=\Sigma_{1}^{2} V_{1} X$. It follows that for arbitrary $k \in \mathbb{N}$, the 252 eigenvector $V$ in (17) can be replaced by

$$
\Sigma_{1}^{2 k} V \Sigma_{1}^{2 k}=\Sigma_{1}^{2 k} V_{1} V_{1}^{T} \Sigma_{1}^{2 k}
$$

253 because

$$
\begin{aligned}
0= & \Sigma_{1}^{2} V_{1}\left(X+X^{T}+Y Y^{T}\right) V_{1}^{T} \Sigma_{1}^{2} \\
= & A_{11}\left(\Sigma_{1}^{2} V_{1} V_{1}^{T} \Sigma_{1}^{2}\right)+\left(\Sigma_{1}^{2} V_{1} V_{1}^{T} \Sigma_{1}^{2}\right) A_{11}^{T} \\
& +N_{11}\left(\Sigma_{1}^{2} V_{1} V_{1}^{T} \Sigma_{1}^{2}\right) N_{11}^{T} .
\end{aligned}
$$

254 Induction leads to

$$
\begin{aligned}
0=A_{11}\left(\Sigma_{1}^{2 k} V_{1} V_{1}^{T} \Sigma_{1}^{2 k}\right)+\left(\Sigma_{1}^{2 k} V_{1} V_{1}^{T} \Sigma_{1}^{2 k}\right) A_{11}^{T} \\
+
\end{aligned}
$$

255 As above, we conclude that $N_{21} \Sigma_{1}^{2 k} V_{1}=0, \tilde{C}_{1} \Sigma_{1}^{2 k} V_{1}=0$, and $256 \tilde{B}_{1}^{T} \Sigma_{1}^{2 k} V_{1}=0$. Multiplying the lower left blocks of (13a) and 257 (13b) with $\Sigma_{1}^{2(k-1)} V_{1}$ and $\Sigma_{1}^{2 k} V_{1}$, respectively, we get

$$
\begin{gathered}
A_{12}^{T} \Sigma_{1}^{2 k-1} V_{1}+\Sigma_{2} A_{21} \Sigma_{1}^{2(k-1)} V_{1}+N_{12}^{T} \Sigma_{1}^{2 k-1} V_{1} Y=0 \\
A_{12}^{T} \Sigma_{1}^{2 k-1} V_{1}+\Sigma_{2}^{-1} A_{21} \Sigma_{1}^{2 k} V_{1}+N_{12}^{T} \Sigma_{1}^{2 k-1} V_{1} Y=0 .
\end{gathered}
$$

258 Hence (after multiplication with $\Sigma_{2}$ ), for all $k \geq 1$, we have

$$
\begin{aligned}
\Sigma_{2}^{2} A_{21} \Sigma_{1}^{2(k-1)} V_{1} & =-\Sigma_{2}\left(A_{12}^{T} \Sigma_{1}^{2 k-1} V_{1}+N_{12}^{T} \Sigma_{1}^{2 k-1} V_{1} Y\right) \\
& =A_{21} \Sigma_{1}^{2 k} V_{1} .
\end{aligned}
$$

259 Applying this identity repeatedly, we get

$$
A_{21} \Sigma_{1}^{2 k} V_{1}=\Sigma_{2}^{2 k} A_{21} V_{1} \text { for all } k \in \mathbb{N} .
$$

260 If $\mu$ is the minimal polynomial of $\Sigma_{1}^{2}$, then $\sigma\left(\Sigma_{1}\right) \cap \sigma\left(\Sigma_{2}\right)=\emptyset$ 261 implies $\operatorname{det} \mu\left(\Sigma_{2}^{2}\right) \neq 0$ and

$$
0=A_{21} \mu\left(\Sigma_{1}^{2}\right) V_{1}=\mu\left(\Sigma_{2}^{2}\right) A_{21} V_{1}
$$

262 whence $A_{21} V_{1}=0$ and also $A_{21} V=0$. Hence we obtain the 263 contradiction (19).

\section{B. Error Estimate}

265 The following theorem has been proven in [19] using LMI266 techniques. Exploiting the stability result in the previous sub267 section, we can give a slightly more compact proof based on 268 the stochastic bounded real lemma, Theorem A.6.

269 Theorem II.4: Let $A$ and $N$ satisfy
Assume furthermore that for $\Sigma=\operatorname{diag}\left(\Sigma_{1}, \Sigma_{2}\right)>0$ with $\Sigma_{2}=270$ $\operatorname{diag}\left(\sigma_{r+1} I, \ldots, \sigma_{\nu} I\right)$ and $\sigma\left(\Sigma_{1}\right) \cap \sigma\left(\Sigma_{2}\right)=\emptyset$, the following 271 Lyapunov inequalities hold:

$$
\begin{gathered}
A^{T} \Sigma+\Sigma A+N^{T} \Sigma N \leq-C^{T} C \\
A^{T} \Sigma^{-1}+\Sigma^{-1} A+N^{T} \Sigma^{-1} N \leq-\Sigma^{-1} B B^{T} \Sigma^{-1} .
\end{gathered}
$$

If $x(0)=0$ and $x_{r}(0)=0$, then for all $T>0$, it holds that

$$
\left\|y-y_{r}\right\|_{L_{w}^{2}([0, T])} \leq 2\left(\sigma_{r+1}+\cdots+\sigma_{\nu}\right)\|u\|_{L_{w}^{2}([0, T])} .
$$

Proof: We adapt a proof for deterministic systems, e.g., 274 [2, Th. 7.9]. In the central argument we treat the case where 275 $\Sigma_{2}=\sigma_{\nu} I$ and show that

$$
\left\|y-y_{\nu-1}\right\|_{L_{w}^{2}[0, T]} \leq 2 \sigma_{\nu}\|u\|_{L_{w}^{2}[0, T]} .
$$

From the left upper blocks of (13a) and (13b), we can see 277 that also

$$
\begin{aligned}
A_{11}^{T} \Sigma_{1}+\Sigma_{1} A_{11}+N_{11}^{T} \Sigma_{1} N_{11} & \leq-C_{1}^{T} C_{1} \\
A_{11}^{T} \Sigma_{1}^{-1}+\Sigma_{1}^{-1} A_{11}+N_{11}^{T} \Sigma_{1}^{-1} N_{11} & \leq-\Sigma_{1}^{-1} B_{1} B_{1}^{T} \Sigma_{1}^{-1} .
\end{aligned}
$$

Hence we can repeat the above argument to remove $\sigma_{\nu-1}, 279$ $\ldots, \sigma_{r+1}$ successively. By the triangle inequality we find that 280

$$
\begin{aligned}
\left\|y-y_{r}\right\|_{L_{w}^{2}[0, T]} & \leq \sum_{j=r}^{\nu-1}\left\|y_{j+1}-y_{j}\right\|_{L_{w}^{2}[0, T]} \\
& \leq 2\left(\sigma_{\nu}+\cdots+\sigma_{r+1}\right)\|u\|_{L_{w}^{2}[0, T]} .
\end{aligned}
$$

which then concludes the proof.

To prove (22), we make use of the stochastic bounded real 282 lemma. In the following let $r=\nu-1$ and consider the error 283 system defined by:

$$
\begin{aligned}
d x_{e} & =A_{e} x_{e} d t+N_{e} x_{e} d w+B_{e} u d t \\
y_{e} & =C_{e} x_{e}=y-y_{r}
\end{aligned}
$$

where

$$
\begin{aligned}
x_{e} & =\left[\begin{array}{l}
x_{1} \\
x_{2} \\
x_{r}
\end{array}\right] \quad A_{e}=\left[\begin{array}{ccc}
A_{11} & A_{12} & 0 \\
A_{21} & A_{22} & 0 \\
0 & 0 & A_{11}
\end{array}\right] \\
N_{e} & =\left[\begin{array}{ccc}
N_{11} & N_{12} & 0 \\
N_{21} & N_{22} & 0 \\
0 & 0 & N_{11}
\end{array}\right] \quad B_{e}=\left[\begin{array}{l}
B_{1} \\
B_{2} \\
B_{1}
\end{array}\right] \\
C_{e} & =\left[\begin{array}{lll}
C_{1} & C_{2} & -C_{1}
\end{array}\right] .
\end{aligned}
$$

Applying the state space transformation 
287 we obtain the transformed system

$$
\begin{aligned}
& \tilde{A}_{e}=S^{-1} A_{e} S=\left[\begin{array}{ccc}
A_{11} & A_{12} & 0 \\
\frac{1}{2} A_{21} & A_{22} & \frac{1}{2} A_{21} \\
0 & A_{12} & A_{11}
\end{array}\right] \\
& \tilde{N}_{e}=S^{-1} N_{e} S=\left[\begin{array}{ccc}
N_{11} & N_{12} & 0 \\
\frac{1}{2} N_{21} & N_{22} & \frac{1}{2} N_{21} \\
0 & N_{12} & N_{11}
\end{array}\right] \\
& \tilde{B}_{e}=S^{-1} B\left[\begin{array}{c}
0 \\
B_{2} \\
2 B_{1}
\end{array}\right] \\
& \tilde{C}_{e}=C_{e} S=\left[\begin{array}{lll}
C_{1} & C_{2} & 0
\end{array}\right] .
\end{aligned}
$$

288 By Theorem A.6, we have $\left\|\mathbb{L}_{e}\right\| \leq 2 \sigma_{\nu}$, if the Riccati inequality

$$
\begin{aligned}
\mathcal{R}_{\sigma_{\nu}}(X)=\tilde{A}_{e}^{T} X+X \tilde{A}_{e}+\tilde{N}_{e}^{T} X \tilde{N}_{e}+\tilde{C}_{e}^{T} \tilde{C}_{e} & \\
& +\frac{1}{4 \sigma_{\nu}^{2}} X \tilde{B}_{e} \tilde{B}_{e}^{T} X \leq 0
\end{aligned}
$$

289 possesses a solution $X \geq 0$. In fact, such a solution is given by 290 the block-diagonal matrix

$$
X=\operatorname{diag}\left(\Sigma_{1}, 2 \Sigma_{2}, \sigma_{\nu}^{2} \Sigma_{1}^{-1}\right)=\operatorname{diag}\left(\Sigma_{1}, 2 \sigma_{\nu} I, \sigma_{\nu}^{2} \Sigma_{1}^{-1}\right)>0 .
$$

291 To verify this, we set $J=\left[\begin{array}{ll}0 & I \\ I & 0\end{array}\right]$ and

$$
M=J\left(A^{T} \Sigma^{-1}+\Sigma^{-1} A+N^{T} \Sigma^{-1} N+\Sigma^{-1} B B^{T} \Sigma^{-1}\right) J
$$

292 where $M \leq 0$ by (13b). Considering all blocks of (13a) and 293 (13b), a straight-forward computation yields

$$
\begin{aligned}
\mathcal{R}_{\sigma_{\nu}}(X)=\left[\begin{array}{cc}
A^{T} \Sigma+\Sigma A+N^{T} \Sigma N+C^{T} C & 0 \\
0 & 0
\end{array}\right] \\
-\frac{\sigma_{\nu}}{2}\left[\begin{array}{c}
N_{21}^{T} \\
0 \\
-N_{21}^{T}
\end{array}\right]\left[\begin{array}{c}
N_{21}^{T} \\
0 \\
-N_{21}^{T}
\end{array}\right]^{T}+\sigma_{\nu}^{2}\left[\begin{array}{ll}
0 & 0 \\
0 & M
\end{array}\right] \leq 0
\end{aligned}
$$

294 which is inequality (23).

295 Example II.5: Let the system $(A, N, B, C)$ and $Q$ be as in 296 Example I.3. The matrix

$$
P=\left[\begin{array}{cc}
1+\sqrt{1-p} & 0 \\
0 & p
\end{array}\right]^{-1}>0, \text { where } 0<p \leq 1
$$

297 satisfies inequality (7b). As in Example I.3, we have $\mathbb{L}_{r}=0$ 298 for the corresponding reduced system of order 1 , so that the 299 truncation error again is $1 / \sqrt{2} a$, independently of $p \in] 0,1]$.

300 On the other hand we have

$$
\sigma_{2}^{2}=\min \sigma(P Q)=\frac{1}{4 a^{2}(1+\sqrt{1-p})} \geq \frac{1}{8 a^{2}}
$$

301 with equality for $p \rightarrow 0$. Theorem II.4 thus gives the sharp error 302 bound $2 \sigma_{2}=1 / \sqrt{2} a$. Note, that there is no $P>0$ satisfying (7b). 303 The previous example illustrates the problem of optimizing 304 over all solutions of inequality (7b).
IV. NUMERICAL EXAMPLES

305

To compare the reduction methods, we need to compute $Q, P 306$ from (6) or (7). Instead of the inequalities (6a), (6b), (7a) we can 307 consider the corresponding equations, for which quite efficient 308 algorithms have been developed recently, e.g., [27]-[30]. These 309 also allow for a low-rank approximation of the solutions. In 310 contrast we cannot replace (7b) by the corresponding equation, 311 because this may not be solvable (see Example II.5). Even 312 worse, we neither have any solvability or uniqueness criteria 313 nor reliable algorithms.

314

Therefore, in general, we have to work with the inequality 315 (7b), which is solvable according to Lemma II.1, but of course 316 not uniquely solvable.

317

In view of our application, we aim at a solution $P$ of $(7 \mathrm{~b}), 318$ so that (some of) the eigenvalues of $P Q$ are particularly small, 319 since they provide the error bound. Choosing a matrix $Y<0320$ and a very small $\varepsilon$ along the lines of the proof of Lemma II.1 321 can be contrary to this aim. Hence some optimization over all 322 solutions of (7b) is required.

Note also that a matrix $P>0$ satisfies (7b), if and only if it 324 satisfies the linear matrix inequality (LMI)

$$
\left[\begin{array}{cc}
P A^{T}+A P+B B^{T} & P N^{T} \\
N P & -P
\end{array}\right] \leq 0 .
$$

Thus, LMI optimal solution techniques are applicable. How- 326 ever, their complexity will be prohibitive for large-scale prob- 327 lems. Therefore further research for alternative methods to 328 solve (7b) adequately is required.

329

By $\mathbb{L}$ and $\mathbb{L}_{r}$, we always denote the original and the $r$-th 330 order approximated system. The stochastic $H^{\infty}$-type norm 331 $\left\|\mathbb{L}-\mathbb{L}_{r}\right\|$ is computed by a binary search of the infimum of all 332 $\gamma$ such that the Riccati inequality (10) is solvable. The latter is 333 solved via a Newton iteration as in [18]. Finally, the Lyapunov 334 equations (2) are solved by preconditioned Krylov subspace 335 methods described in [27].

Unfortunately, for small $\gamma$, i.e., for small approximation 337 errors, this method of computing the error runs into numerical 338 problems, because (10) contains the term $\gamma^{-2}$. This apparently 339 leads to cancellation phenomena in the Newton iteration, if, 340 e.g., $\gamma<10^{-7}$. Therefore we mainly concentrate on cases 341 where the error is larger, i.e., we make $r$ sufficiently small. $\quad 342$

\section{A. Type II Can be Better Than Type I}

In many examples we observe that type II reduction gives a 344 valid error bound, but the approximation error still is better with 345 type I. This, however, is not always true, as the example

346

$$
\left(A, N, B, C^{T}\right)=\left(\left[\begin{array}{cc}
-1 & 1 \\
0 & -1
\end{array}\right],\left[\begin{array}{ll}
0 & 0 \\
1 & 0
\end{array}\right],\left[\begin{array}{l}
0 \\
3
\end{array}\right],\left[\begin{array}{l}
3 \\
0
\end{array}\right]\right)
$$

shows. It can easily be verified that the type I Lyapunov 347 equations (6) are solved by

$$
Q=\left[\begin{array}{ll}
6 & 3 \\
3 & 3
\end{array}\right] \quad P=\left[\begin{array}{ll}
3 & 3 \\
3 & 6
\end{array}\right] .
$$

The type II inequalities (7) are, e.g., solved by

$$
Q=\left[\begin{array}{ll}
6 & 3 \\
3 & 3
\end{array}\right] \quad P=\left[\begin{array}{rr}
8 & 0 \\
0 & 12
\end{array}\right]
$$


TABLE I

ERROR BOUNDS AND APPROXIMATION ERRORS FOR BOTH TYPES

\begin{tabular}{l||c|c}
\hline & $\sigma_{2}$ & $\left\|\mathbb{L}-\mathbb{L}_{1}\right\|$ \\
\hline I & 2.4853 & 3.9647 \\
\hline II & 6.9282 & 3.5614 \\
\hline
\end{tabular}

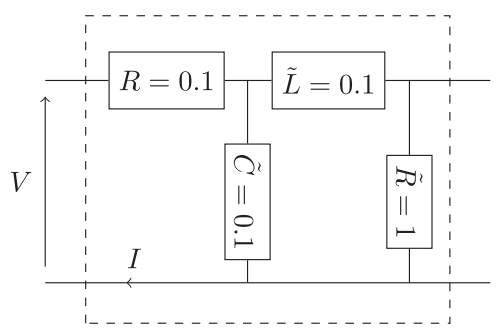

Fig. 1. Section of ladder network from [31].

350 If we reduce to order $r=1$, the type I approximation error is 351 larger than both the truncated singular value and the type II 352 approximation error; see Table I.

\section{B. Electrical Ladder Network With Perturbed Inductance}

354 As our first example with a physical background, we take 355 up the electrical ladder network described in [31], consisting of $356 n / 2$ sections with a capacitor $\tilde{C}$, inductor $\tilde{L}$ and two resistors $357 R$ and $\tilde{R}$ as depicted in Fig. 1 .

358 But following, e.g., [32], we assume that the inductance $\tilde{L}$ is 359 subject to stochastic perturbations. For simplicity, we replace the 360 inverse $\tilde{L}^{-1}$ formally by $L^{-1}+\dot{w}$ in all sections. Here $L=0.1$ 361 and $\dot{w}$ is white noise of a certain intensity $\sigma$, where we set $\sigma=1$, 362 e.g., for $n=6$, we have the system matrices

$$
\begin{aligned}
& A=\left[\begin{array}{cccccc}
\frac{-1}{\tilde{C} R} & \frac{-1}{\tilde{C}} & 0 & 0 & 0 & 0 \\
\frac{1}{L} & \frac{-R \tilde{R}}{L(R+R)} & \frac{-\tilde{R}}{L(R+R)} & 0 & 0 & 0 \\
0 & \frac{\tilde{R}}{\tilde{C}(R+\tilde{R})} & \frac{-1}{\tilde{C}(R+\tilde{R})} & \frac{-1}{C} & 0 & 0 \\
0 & 0 & \frac{1}{L} & \frac{-R \tilde{R}}{L(R+\tilde{R})} & \frac{-\tilde{R}}{L(R+\tilde{R})} & 0 \\
0 & 0 & 0 & \frac{\tilde{R}}{\tilde{C}(R+\bar{R})} & \frac{-1}{\tilde{C}(R+\tilde{R})} & \frac{-1}{\tilde{C}} \\
0 & 0 & 0 & 0 & \frac{1}{L} & \frac{-\tilde{R}}{L}
\end{array}\right] \\
& N= \\
& B=\left[\begin{array}{cccccccc}
0 & 0 & 0 & 0 & 0 & 0 \\
1 & \frac{-R \tilde{R}}{R+\tilde{R}} & \frac{-\tilde{R}}{R+\tilde{R}} & 0 & 0 & 0 \\
0 & 0 & 0 & 0 & 0 & 0 \\
0 & 0 & 1 & \frac{-R \tilde{R}}{R+\tilde{R}} & \frac{-\tilde{R}}{R+\tilde{R}} & 0 \\
0 & 0 & 0 & 0 & 0 & 0 \\
0 & 0 & 0 & 0 & 1 & -\tilde{R}
\end{array}\right] \\
& C=\left[\begin{array}{cccccccc}
\frac{1}{\tilde{C} R} & 0 & 0 & 0 & 0 & 0 & 0
\end{array}\right]^{T}
\end{aligned}
$$

363 For larger $n$, the band structure of $A$ and $N$ is extended 364 periodically. To see the behavior of our two methods, we reduce 365 from order $n=20$ to the orders $r=1,3,5, \ldots, 19$, and com366 pute both the theoretical bounds and the actual approximation 367 errors in the $H^{\infty}$-norm; see Fig. 2.

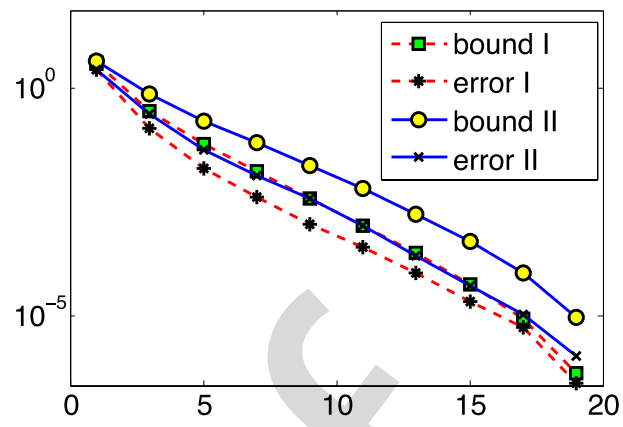

Fig. 2. In this example, for both types the bounds hold, and for all reduced orders, type I gives a smaller $H^{\infty}$-error than type II.
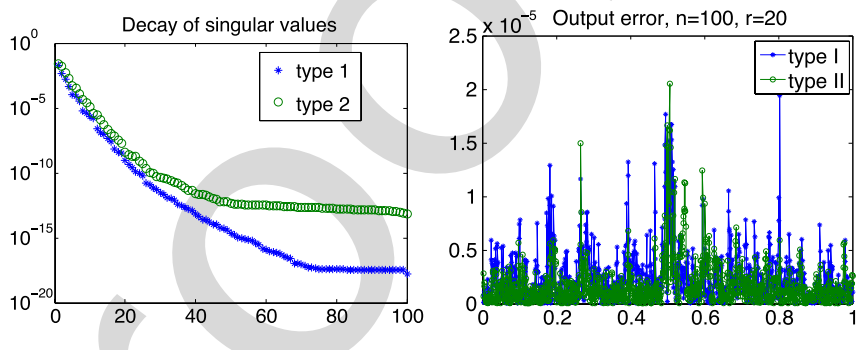

Fig. 3. Comparison of singular values and relative output error

\section{Heat Transfer Problem}

As another example we consider a stochastic modification of 369 the heat transfer problem described in [14]. On the unit square 370 $\Omega=[0,1]^{2}$, the heat equation $x_{t}=\Delta x$ is given with Dirichlet 371 condition $x=u_{j}, j=1,2,3$, on three of the boundary edges 372 and a stochastic Robin condition $n \cdot \nabla x=(1 / 2+\dot{w}) x$ on the 373 fourth edge (where $\dot{w}$ stands for white noise). A standard five- 374 point finite-difference discretization on a $10 \times 10$ grid leads 375 to a modified Poisson matrix $A \in \mathbb{R}^{100 \times 100}$ and corresponding 376 matrices $N \in \mathbb{R}^{100 \times 100}$ and $B \in \mathbb{R}^{100 \times 3}$. We use the input 377

$$
u \equiv\left[\begin{array}{l}
1 \\
1 \\
1
\end{array}\right]
$$

and choose the average temperature as the output, i.e., $C=378$ $(1 / 100)[1, \ldots, 1]$. We apply balanced truncation of type I 379 and type II. For type II, an LMI-solver (MATLAB function 380 mincx) is used to compute $P$ as a solution of the LMI (24) 381 which minimizes trace $P$ or trace $P Q$.

In the following figure (Fig. 3), we compare the reduced 383 systems of order $r=20$ for both types. The left diagram shows 384 the decay of the singular values. Since the LMI-solver was 385 called with tolerance level $10^{-9}$, only the first about 25 singular 386 values for type II have the correct order of magnitude. In this 387 region, the decay for both types is roughly linear. Some analysis 388 of this behavior for type I has been carried out in [28]. For 389 type II, so far no theoretical results are available.

The diagram on the right displays the approximation error 391 $\left\|y(t)-y_{r}(t)\right\|$ over a given time interval. For both types it has 392 the same order of magnitude. In fact, for many examples we 393 have observed both methods to yield very similar results. $\quad 394$

The estimated error norm $\sum_{j=r+1}^{n} \sigma_{j}$ and the actual approx- 395 imation error $\left\|\mathbb{L}-\mathbb{L}_{10}\right\|$ are given in Table II. 
TABLE II

ERROR BOUNDS AND APPROXIMATION ERRORS FOR BOTH TYPES

\begin{tabular}{l||c|c||c|c}
\hline & $\sum_{j=11}^{100} \sigma_{j}$ & $\left\|\mathbb{L}-\mathbb{L}_{10}\right\|$ & $\sum_{j=21}^{100} \sigma_{j}$ & $\left\|\mathbb{L}-\mathbb{L}_{20}\right\|$ \\
\hline I & $4.66 e-06$ & $9.30 e-06$ & $2.00 e-09$ & $9.65 e-09$ \\
\hline II & $1.75 e-05$ & $4.83 e-06$ & $1.72 e-08$ & $9.70 e-09$ \\
\hline
\end{tabular}

TABLE III

COMPARISON OF BOTH REDUCTION METHODS

\begin{tabular}{l||c|c}
\hline Type & I & II \\
\hline \hline Def. of $P, Q$ & $(6)$ & (7) \\
\hline Stability? & Yes, [24] & Yes, Thm. II.2 \\
\hline$H^{2}$-bound? & Yes, [20] & Yes, [33] \\
\hline$H^{\infty}$-bound? & No, Ex. I.3 & Yes, Thm. II.4 or [19] \\
\hline comput. cost & medium & high (via LMI) \\
\hline
\end{tabular}

397 As we can see, the upper error bound fails for type I, but is 398 correct for type II. Nevertheless, judging from the $H^{\infty}$ error, 399 neither of the types seems to be preferable over the other.

\section{D. Summary}

401 Clearly, higher dimensional examples are required to get 402 more insight. To this end, a more sophisticated method for the 403 solution of (24) is needed. With general-purpose LMI-software 404 on a standard Laptop, we hardly got higher than $n=100$.

\section{COMPARISON}

406 Table III summarizes properties of our two methods.

407 As long as efficient algorithms for the solution of (7b) are not 408 available, practical evidence favors to use the type I method in 409 applications. Although there is no strict $H^{\infty}$-type error bound 410 for this case, in most examples the decay of singular values still 411 roughly indicates the decay of the approximation error.

\section{VI. CONCLUSIONS AND FUTURE WORK}

413 We have discussed two ways of generalizing balanced trun414 cation for stochastic linear systems. The main theoretical con415 tributions of this paper are the preservation of asymptotic 416 stability for type II balanced truncation proved in Theorem II.2 417 and the new proof of the $H^{\infty}$ error bound in Theorem II.4. 418 The efficient solution of the matrix inequality (7b) is an open 419 issue and requires further research. The same is true for the 420 computation of the stochastic $H^{\infty}$-norm. Moreover, we are still 421 looking for adequate interpretations of our approaches, e.g., in 422 terms of energy minimization or Hankel operators. We hope to 423 trigger some research in this direction.

424

\section{APPENDIX A}

\section{ASYMPTOTIC MEAN SQUARE STABILITy}

Consider the stochastic linear system of Itô-type

$$
d x=A x d t+N x d w
$$

427 where $w=(w(t))_{t \in \mathbb{R}_{+}}$is a zero-mean real Wiener process on a 428 probability space $(\Omega, \mathcal{F}, \mu)$ with respect to an increasing family $429\left(\mathcal{F}_{t}\right)_{t \in \mathbb{R}_{+}}$of $\sigma$-algebras $\mathcal{F}_{t} \subset \mathcal{F}$ (e.g., [25], [26]).
Let $L_{w}^{2}\left(\mathbb{R}_{+}, \mathbb{R}^{q}\right)$ denote the corresponding space of nonan- 430 ticipating stochastic processes $v$ with values in $\mathbb{R}^{q}$ and norm $\quad 431$

$$
\|v(\cdot)\|_{L_{w}^{2}}^{2}:=\mathcal{E}\left(\int_{0}^{\infty}\|v(t)\|^{2} d t\right)<\infty
$$

where $\mathcal{E}$ denotes expectation. For initial data $x(0)=x_{0}$, the 432 solution can be written as $x(t)=\Phi(t) x_{0}$ with the fundamental 433 matrix solution $\Phi(t)$, satisfying $\Phi(0)=I$

By definition, system (25) is asymptotically mean-square- 435 stable, if $\mathcal{E}\left(\|x(t)\|^{2}\right) \stackrel{t \rightarrow \infty}{\longrightarrow} 0$, for all initial conditions $x_{0}$. In this 436 case, for simplicity, we also call the pair $(A, N)$ asymptotically 437 mean-square stable.

438

We have the following version of Lyapunov's matrix 439 theorem; see [23]. Here $\otimes$ denotes the Kronecker product. $\quad 440$

Theorem A.1: The following are equivalent.

(i) System (25) is asymptotically mean-square stable.

(ii) $\max \{\Re \lambda \| \lambda \in \sigma(A \otimes I+I \otimes A+N \otimes N)\}<0$

(iii) $\exists Y>0$ : $\exists X>0$ : $A^{T} X+X A+N^{T} X N=-Y$

(iv) $\forall Y>0: \exists X>0: A^{T} X+X A+N^{T} X N=-Y$

(v) $\forall Y \geq 0: \exists X \geq 0: A^{T} X+X A+N^{T} X N=-Y$

442

443

444

445

446

Remark A.2: The theorem (like all other results in this paper) 448 carries over to systems

$$
d x=A x d t+\sum_{j=1}^{k} N_{j} x d w_{j}
$$

with more than one noise term, and many more equivalent 450 criteria can be provided; see, e.g., [34] or [18, Th. 3.6.1]. 451

The following theorem does not require any stability assump- 452 tions (see [18, Th. 3.2.3]). It is central in the analysis of mean- 453 square stability.

Theorem A.3: Let

$$
\alpha=\max \{\Re \lambda \mid \lambda \in \sigma(A \otimes I+I \otimes A+N \otimes N)\} .
$$

Then there exists a nonnegative definite matrix $V \neq 0$, so that 456

$$
\left(\mathcal{L}_{A}^{*}+\Pi_{N}^{*}\right)(V)=A V+V A^{T}+N V N^{T}=\alpha V .
$$

We also note a simple consequence of this theorem [24, 457 Cor. 3.2]. Here $\langle Y, V\rangle=\operatorname{trace}(Y V)$ is the Frobenius inner 458 product for symmetric matrices.

Corollary A.4: Let $\alpha, V$ as in the theorem. For given $Y \geq 0460$ assume that

$$
\exists X>0: \mathcal{L}_{A}(X)+\Pi_{N}(X) \leq-Y .
$$

Then $\alpha \leq 0$. Moreover, if $\alpha=0$ then $Y V=V Y=0$.

\section{APPENDIX B}

\section{Stochastic Bounded REAL LEMMA}

Now let us consider system (5) with input $u$ and output $y .465$ If $(A, N)$ is asymptotically mean-square stable, then (5) de- 466 fines an input output operator $\mathbb{L}: u \mapsto y$ from $L_{w}^{2}\left(\mathbb{R}, \mathbb{R}^{m}\right)$ to 467 $L_{w}^{2}\left(\mathbb{R}, \mathbb{R}^{p}\right)$, see [17]. By $\|\mathbb{L}\|$ we denote the induced operator 468 norm, which is an analogue of the deterministic $H^{\infty}$-norm. It 469 can be characterized by the stochastic bounded real lemma. $\quad 470$ 
(iii) There exists a positive definite solution $X>0$ to the Riccati inequality

$$
A^{T} X+X A+N^{T} X N+C^{T} C+\gamma^{-2} X B B^{T} X<0
$$

479 We have stated the obviously equivalent formulations (ii) and 480 (iii) to avoid confusion arising from different formulations 481 in the literature. Under additional assumptions also nonstrict 482 versions can be formulated. The following sufficient criterion 483 is given in [18, Cor. 2.2.3] (where also the signs are changed). 484 Unlike in the previous theorem, here asymptotic mean-square 485 stability is assumed at the outset.

486 Theorem A.6: Assume that (25) is asymptotically stable in 487 mean-square. If there exists a nonnegative definite matrix $X \geq 0$, 488 satisfying

$$
A^{T} X+X A+N^{T} X N+C^{T} C+\gamma^{-2} X B B^{T} X \leq 0
$$

489 then $\|\mathbb{L}\| \leq \gamma$.

490

491

492 Definition A.7: Consider system (5). A vector $v \in \mathbb{R}^{n}$ is 493 called unobservable, if the initial condition $x(0)=v$ with $u \equiv 0$ 494 produces the output $y \equiv 0$. The vector $v$ is called unreachable, 495 if $x(t) \neq v$ for all $t>0$ and any solution with initial value $496 x(0)=0$ and arbitrary input $u$.

497 If $(A, N)$ is asymptotically mean-square stable, then (see [14, 498 Th. 3.1]) the unobservable and the unreachable subspace can be 499 characterized as the kernels of $Q$ and $P$ defined by

$$
\begin{aligned}
& A^{T} Q+Q A+N^{T} Q N=-C^{T} C \\
& A P+P A^{T}+N P N^{T}=-B B^{T} .
\end{aligned}
$$

509 Theorem A.8: A state $v$ is

502 (a) unobservable, if and only if $Q v=0$.

503 (b) unreachable, if and only if $P v=0$.

504 In particular, the system is observable and reachable, if and only 505 if $Q>0$ and $P>0$.

\section{REFERENCES}

[1] G. Obinata and B. Anderson, Model Reduction for Control System Design. New York, NY, USA: Springer, 2001.

[2] A. C. Antoulas, Approximation of Large-Scale Dynamical Systems, ser. Advances in Design and Control. Philadelphia, PA, USA: Soc. Industrial and Appl. Math., 2005, vol. 6.

[3] P. Benner, V. Mehrmann, and D. C. Sorensen, Eds., Dimension Reduction of Large-Scale Systems, ser. Lecture Notes in Computational Science and Engineering, vol. 45. New York, NY, USA: Springer-Verlag, 2005.

[4] W. H. Schilders, H. A. van der Vorst, and J. Rommes, Eds., Model Order Reduction: Theory, Research Aspects and Applications, ser. Mathematics in Industry, vol. 13. Berlin, Germany: Springer-Verlag, 2008.

[5] U. Baur, P. Benner, and L. Feng, "Model order reduction for linear and nonlinear systems: A system-theoretic perspective," Arch. Comput. Method. E., vol. 21, no. 4, pp. 331-358, 2014.
[6] B. C. Moore, "Principal component analysis in linear systems: controlla- 521 bility, observability, and model reduction," IEEE Trans. Autom. Control, 522 vol. AC-26, pp. 17-32, 1981.

[7] L. Pernebo and L. M. Silverman, "Model reduction via balanced state 524 space representations," IEEE Trans. Autom. Control, vol. AC-27, no. 2, 525 pp. 382-387, 1982.

[8] T. Stykel, "Analysis and numerical solution of generalized Lyapunov 527 equations," Ph.D. dissertation, Tech. Univ. Berlin, Berlin, Germany 2002. 528

[9] A. Shokoohi, L. M. Silverman, and P. M. Van Dooren, "Linear time- 529 variable systems: balancing and model reduction," IEEE Trans. Autom. 530 Control, vol. AC-28, no. 8, pp. 810-822, 1983.

[10] E. Verriest and T. Kailath, "On generalized balanced realizations," IEEE 532 Trans. Autom. Control, vol. AC-28, no. 8, pp. 833-844, 1983.

[11] H. Sandberg and A. Rantzer, "Balanced truncation of linear time-varying 534 systems," IEEE Trans. Autom. Control, vol. 49, no. 2, pp. 217-229, 535 Feb. 2004.

[12] S. A. Al-Baiyat, M. Bettayeb, and U. M. Al-Saggaf, "New model reduc- 537 tion scheme for bilinear systems," Int. J. Syst. Sci., vol. 25, pp. 1631-1642, 538 1994.

[13] W. S. Gray and J. Mesko, "Energy functions and algebraic Gramians 540 for bilinear systems," in Proc. 4th IFAC Nonlinear Control Syst. Design 541 Symp., Enschede, The Netherlands, 1998, pp. 103-108. 542

[14] P. Benner and T. Damm, "Lyapunov equations, energy functionals, and 543 model order reduction of bilinear and stochastic systems," SIAM J. 544 Control Optim., vol. 49, no. 2, pp. 686-711, 2011.

[15] J. M. A. Scherpen, "Balancing for nonlinear systems," Syst. Control Lett., 546 vol. 21 , no. 2 , pp. $143-153,1993$.

[16] W. M. Wonham, "Random differential equations in control theory," in 548 Probabilstic Methods in Applied Mathematics, A. T. Bharucha-Reid, Ed., 549 vol. 2. New York, NY, USA: Academic, 1970, pp. 131-212. 550

[17] D. Hinrichsen and A. J. Pritchard, "Stochastic $H_{\infty}$," SIAM J. Control 551 Optim., vol. 36, no. 5, pp. 1504-1538, 1998.

[18] T. Damm, Rational Matrix Equations in Stochastic Control, ser. Lec- 553 ture Notes in Control and Information Sciences. New York, NY, USA: 554 Springer, 2004, no. 297.

[19] T. Damm and P. Benner, "Balanced truncation for stochastic linear sys- 556 tems with guaranteed error bound," in Proc. MTNS-2014, Groningen, 557 The Netherlands, 2014, pp. 1492-1497.

[20] P. Benner and M. Redmann, "Model reduction for stochastic systems," 559 Stochastic Partial Diff. Eq.: Anal. Comput., vol. 3, no. 3, pp. 291-338, 560 2015.

[21] F. R. Gantmacher, The Theory of Matrices, vol. 2. New York, NY, USA: 562 Chelsea, 1959.

[22] D. L. Kleinman, "On the stability of linear stochastic systems," IEEE 564 Trans. Autom. Control, vol. AC-14, pp. 429-430, 1969.

[23] R. Z. Khasminskij, Stochastic Stability of Differential Equations. 566 Alphen aan den Rijn, NL: Sijthoff \& Noordhoff, 1980.

[24] P. Benner, T. Damm, M. Redmann, and Y. Rocio Rodriguez Cruz, "Pos- 568 itive operators and stable truncation," Linear Algebra Appl., vol. 498, 569 Jun. 2016, pp. 74-87.

[25] L. Arnold, Stochastic Differential Equations: Theory and Applications. 571 Translation. New York, NY, USA: Wiley, $1974 . \quad 572$

[26] B. Oeksendal, Stochastic Differential Equations, 5th ed. Berlin, 573 Germany: Springer-Verlag, 1998.

[27] T. Damm, "Direct methods and ADI-preconditioned Krylov subspace 575 methods for generalized Lyapunov equations," Numer. Linear Alg. Appl., 576 vol. 15, no. 9, pp. 853-871, 2008.

[28] P. Benner and T. Breiten, "Low rank methods for a class of generalized 578 Lyapunov equations and related issues," Numer. Math., vol. 124, no. 3, 579 pp. 441-470, 2013.

[29] D. Kressner and P. Sirković, "Greedy low-rank methods for solving 581 general linear matrix equations," Numer. Alg. and High-Performance 582 Comput. (ANCHP), Math. Inst. Comput. Sci. Eng. (MATHICSE), École 583 Polytechnique Fédérale de Lausanne, Lausanne, Switzerland, 2014. 584

[30] S. Shank, V. Simoncini, and D. Szyld, "Efficient low-rank solu- 585 tions of generalized Lyapunov equations," Dept. Math., Temple Univ., 586 Philadelphia, PA, USA, 2014.

[31] S. Gugercin and A. Antoulas, "A survey of model reduction by bal- 588 anced truncation and some new results," Int. J. Control, vol. 77, no. 8, 589 pp. 748-766, 2004

590

[32] V. A. Ugrinovskii and I. R. Petersen, "Absolute stabilization and minimax 591 optimal control of uncertain systems with stochastic uncertainty," SIAM 592 J. Control Optim., vol. 37, no. 4, pp. 1089-1122, 1999.

[33] M. Redmann and P. Benner, "An $\mathcal{H}_{2}$-type error bound for balancing- 594 related model order reduction of linear systems with Lévy noise," Max 595 Planck Inst., Magdeburg, Germany, 2015. 596

[34] H. Schneider, "Positive operators and an inertia theorem," Numer. Math., 597 vol. 7, pp. 11-17, 1965.

\section{AQ2




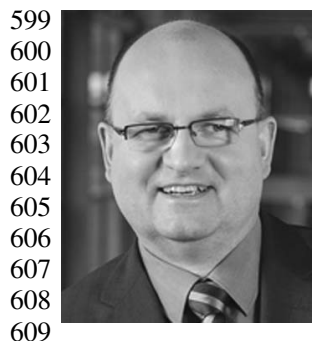

Peter Benner received the Diplom in mathematics from the RWTH Aachen, Aachen, Germany, in 1993. From 1993 to 1997, he worked on his Ph.D. at the University of Kansas, Lawrence, KS USA, and the TU Chemnitz-Zwickau, Germany, where he received the Ph.D. degree in February 1997. In 2001, he received the Habilitation in Mathematics from the University of Bremen, Bremen, Germany, where he held an Assistant Professor position from 1997 to 2001 .

After spending a term as a Visiting Associate 610 Professor at TU Hamburg-Harburg, Germany, he was a Lecturer in mathematics 611 at TU Berlin 2001-203. Since 2003, he has been a Professor for mathematics 612 in industry and technology at TU Chemnitz. In 2010, he was appointed as 613 one of the four directors of the Max Planck Institute for Dynamics of Com614 plex Technical Systems, Magdeburg, Germany. Since 2011, he has also been 615 an Honorary Professor at the Otto-von-Guericke University of Magdeburg. 616 He was named Distinguished Professor at Shanghai University in 2015. His 617 research interests are in the areas of scientific computing, numerical mathemat618 ics, systems theory, and optimal control. A particular emphasis has been on 619 applying methods from numerical linear algebra and matrix theory in systems 620 and control theory. Recent research focuses on numerical methods for optimal 621 control of systems modeled by evolution equations (PDEs, DAEs, SPDEs), 622 model order reduction, preconditioning in optimal control and UQ problems, 623 and Krylov subspace methods for structured or quadratic eigenproblems. 624 Research in all these areas is accompanied by the development of algo625 rithms and mathematical software suitable for modern and high-performance 626 computer architectures.

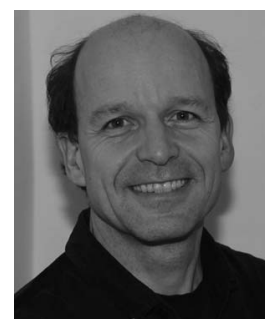

Tobias Damm received the diploma in mathemat- 627 ics from the University of Würzburg, Würzburg, 628 Germany, in 1996 and the doctoral degree from the 629 University of Bremen, Bremen, Germany, in 2002. 630

From 2002 to 2006 he held postdoctoral positions 631 at TU München and TU Braunschweig. He was 632 appointed Junior Professor at the TU Kaiserslautern 633 in 2006 and Associate Professor at the University 634 of Bayreuth in 2011. Since 2012 he has been a 635 Professor for systems and control theory at the TU 636 Kaiserslautern, Kaiserslautern, Germany. His re- 637 search interests encompass stochastic control systems, matrix equations, and 638 numerical methods.

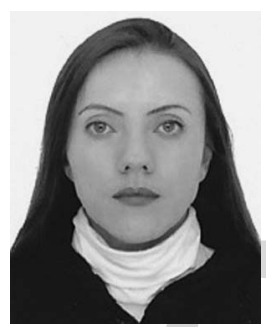

Yolanda Rocio Rodriguez Cruz received the B.Sc. 640 degree in physics from the National University of 641 Colombia, Bogotá, Colombia, in 2004, and the 642 M.Sc. degree in industrial mathematics from the TU 643 Kaiserslautern, Kaiserslautern, Germany, in 2008. 644

From 2009 to 2010, she was a Lecturer at the 645 District University Francisco José de Caldas, Bogotá, 646 Colombia. In the intersemestral period in 2011, she 647 was Lecturer at the National University of Colombia. 648 From 2011 to 2013, she was a Lecturer at the Univer- 649 sity of Bogotá Jorge Tadeo Lozano, Bogotá, Colombia. 650 She is currently a Ph.D. researcher at the TU Kaiserslautern. Her research has 651 been concerned with model reduction for stochastic and bilinear systems. 


\section{AUTHOR QUERIES}

\section{AUTHOR PLEASE ANSWER ALL QUERIES}

$\mathrm{AQ} 1$ = "infima" ok?

AQ2 = Please provide department name in Ref. [8].

END OF ALL QUERIES 


\title{
- Dual Pairs of Generalized Lyapunov Inequalities and Balanced Truncation of Stochastic Linear Systems
}

\author{
Peter Benner, Tobias Damm, and Yolanda Rocio Rodriguez Cruz
}

\begin{abstract}
4 Abstract-We consider two approaches to balanced truncation 5 of stochastic linear systems, which follow from different general6 izations of the reachability Gramian of deterministic systems. Both 7 preserve mean-square asymptotic stability, but only the second 8 leads to a stochastic $\boldsymbol{H}^{\infty}$-type bound for the approximation error 9 of the truncated system.
\end{abstract}

10 Index Terms-Asymptotic mean square stability, balanced trun11 cation, generalized Lyapunov equation, model order reduction, 12 stochastic linear system.

\section{3}

\section{INTRODUCTION}

"O PTIMIZATION and (feedback) control of dynamical systems is often computationally infeasible for high dimen17 sional plant models. Therefore, one tries to reduce the order of 18 the system, so that the input-output mapping is still computable 19 with sufficient accuracy, but at considerably smaller cost than 20 for the original system [1]-[5]. To guarantee the desired accu21 racy, computable error bounds are required. Moreover, system 22 properties which are relevant in the context of control system 23 design like asymptotic stability need to be preserved. It has 24 long been known that for linear time-invariant (LTI) systems the 25 method of balanced truncation preserves asymptotic stability 26 and provides an error bound for the $L^{2}$-induced input-output 27 norm, i.e., the $H^{\infty}$-norm of the associated transfer function; 28 see [6], [7]. When considering model order reduction of more 29 general system classes, it is natural to try to extend this ap30 proach. This has been worked out for descriptor systems in 31 [8], for time-varying systems in [9]-[11], for bilinear systems 32 in [12]-[14] and general nonlinear systems, e.g., in [15]. Yet 33 another generalization of LTI systems is obtained considering 34 dynamics driven by noise processes. This leads to the class of 35 stochastic systems, which have been considered in a system 36 theoretic context, e.g., in [16]-[18]. Quite recently, balanced 37 truncation has also been described for linear stochastic systems 38 of Itô type in [14], [19], and [20]. Already the formulation of 39 the method leads to two different variants that are equivalent 40 in the deterministic case, but not so for stochastic systems. It 41 is natural to ask which of the above-mentioned properties of

Manuscript received December 18, 2015; accepted April 25, 2016. Recommended by Associate Editor J. Imura.

P. Benner is with Max Planck Institute for Dynamics of Complex Technical Systems, 39106 Magdeburg, Germany (e-mail: benner@mpi-magdeburg. mpg.de).

T. Damm and Y. R. Rodriguez Cruz are with the Department of Mathematics, University of Kaiserslautern, 67663 Kaiserslautern, Germany (e-mail: damm@ mathematik.uni-kl.de; rodrigue@ mathematik.uni-kl.de).

Color versions of one or more of the figures in this paper are available online at http://ieeexplore.ieee.org.

Digital Object Identifier 10.1109/TAC.2016.2572881 balanced truncation also hold for these variants. The aim of this 42 paper is to answer this question.

Let us recapitulate balanced truncation for linear control 44 systems of the form

$$
\dot{x}=A x+B u \quad y=C x \quad \sigma(A) \subset \mathbb{C}_{-} .
$$

Here $A \in \mathbb{R}^{n \times n}, B \in \mathbb{R}^{n \times m}, C \in \mathbb{R}^{p \times n}$, and $x(t) \in \mathbb{R}^{n}, y(t) \in 46$ $\mathbb{R}^{p}$ and $u(t) \in \mathbb{R}^{m}$ are the state, output, and input of the system, 47 respectively. Moreover $\sigma(A)$ denotes the spectrum of $A$ and $\mathbb{C}_{-} 48$ the open left half complex plane. Let

$$
\mathcal{L}_{A}: X \mapsto A^{T} X+X A
$$

denote the Lyapunov operator and

$$
\mathcal{L}_{A}^{*}: X \mapsto A X+X A^{T}
$$

its adjoint with respect to the Frobenius inner product $\langle Z, Y\rangle=51$ $\operatorname{trace}\left(Y^{T} Z\right)$. Then $\sigma(A) \subset \mathbb{C}_{-}$if and only if there exists a posi- 52 tive definite solution $X$ of the Lyapunov inequality $\mathcal{L}_{A}(X)<0,53$ by Lyapunov's classical stability theorem, see, e.g., [21]. 54

Balanced truncation means truncating a balanced realization. 55 This realization is obtained by a state space transformation 56 computed from the Gramians $P$ and $Q$, which solve the dual 57 pair of Lyapunov equations

$$
\begin{aligned}
& \mathcal{L}_{A}(Q)=A^{T} Q+Q A=-C^{T} C \\
& \mathcal{L}_{A}^{*}(P)=A P+P A^{T}=-B B^{T}
\end{aligned}
$$

or more generally the inequalities

$$
\mathcal{L}_{A}(Q) \leq-C^{T} C \quad \mathcal{L}_{A}^{*}(P) \leq-B B^{T} .
$$

These (in)equalities are essential in the characterization of 60 stability, controllability and observability of system (1). If 61 $\operatorname{det} P \neq 0$, the inequalities (3) can be written as

$$
\begin{aligned}
\mathcal{L}_{A}(Q) & \leq-C^{T} C \\
\mathcal{L}_{A}\left(P^{-1}\right) & =P^{-1} A+A^{T} P^{-1} \leq-P^{-1} B B^{T} P^{-1} .
\end{aligned}
$$

In the present paper we discuss extensions of (3) and (4) for 63 stochastic linear systems.

As indicated above, the equivalent formulations (3) and (4) 65 lead to different generalizations, if we consider Itô-type sto- 66 chastic systems of the form

$$
d x=A x d t+N x d w+B u d t, \quad y=C x
$$

where $A, B, C$ are as in (1) and $N \in \mathbb{R}^{n \times n}$. System (5) is 68 asymptotically mean-square stable (e.g., [18], [22], [23]), if and 69 
70 only if there exists a positive definite solution $X$ of the gener71 alized Lyapunov inequality

$$
\left(\mathcal{L}_{A}+\Pi_{N}\right)(X)=A^{T} X+X A+N^{T} X N<0 .
$$

72 Here $\Pi_{N}: X \mapsto N^{T} X N$ and $\Pi_{N}^{*}: X \mapsto N X N^{T}$. This sta73 bility criterion indicates that in the stochastic context, the 74 generalized Lyapunov operator $\mathcal{L}_{A}+\Pi_{N}$ takes over the role 75 of $\mathcal{L}_{A}$. Substituting $\mathcal{L}_{A}$ by $\mathcal{L}_{A}+\Pi_{N}$ in (3) and (4), we obtain 76 two different dual pairs of generalized Lyapunov inequalities. 77 We call them type I

$$
\begin{aligned}
\left(\mathcal{L}_{A}+\Pi_{N}\right)(Q) & =A^{T} Q+Q A+N^{T} Q N \leq-C^{T} C \\
\left(\mathcal{L}_{A}+\Pi_{N}\right)^{*}(P) & =A P+P A^{T}+N P N^{T} \leq-B B^{T}
\end{aligned}
$$

78 and type II

$$
\begin{aligned}
\left(\mathcal{L}_{A}+\Pi_{N}\right)(Q) & =A^{T} Q+Q A+N^{T} Q N \\
& \leq-C^{T} C \\
\left(\mathcal{L}_{A}+\Pi_{N}\right)\left(P^{-1}\right) & =A^{T} P^{-1}+P^{-1} A+N^{T} P^{-1} N \\
& \leq-P^{-1} B B^{T} P^{-1} .
\end{aligned}
$$

79 Note that (6) corresponds to (3) in the sense that $\mathcal{L}_{A}^{*}(P)$ has 80 been replaced by $\left(\mathcal{L}_{A}+\Pi_{N}\right)^{*}(P)$, while (7) corresponds to 81 (4), where $\mathcal{L}_{A}\left(P^{-1}\right)$ has been replaced by $\left(\mathcal{L}_{A}+\Pi_{N}\right)\left(P^{-1}\right)$. 82 In general (if $N$ and $P$ do not commute), the inequalities (6b) 83 and (7b) are not equivalent. At first glance it is not clear which 84 generalization is more appropriate.

85 If the system is asymptotically mean-square stable, then 86 for both types there are solutions $Q, P>0$. By a suitable 87 state space-transformation, it is possible to balance the system 88 such that $Q=P=\Sigma>0$ is diagonal. Consequently, the usual 89 procedure of balanced truncation can be applied to reduce the 90 order of (5). For simplicity, let us refer to this as type I or type II 91 balanced truncation.

92 Under natural assumptions, this reduction preserves mean93 square asymptotic stability. For type I, this nontrivial fact has 94 been proven in [24]. Moreover, in [20], an $H^{2}$-error bound 95 has been provided. However, different from the deterministic 96 case, there is no $H^{\infty}$-type error bound in terms of the truncated 97 entries in $\Sigma$. This will be shown in Example I.3.

98 In contrast, for type II, an $H^{\infty}$-type error bound has been 99 obtained in [19]. In the present paper, as one of our main 100 contributions, we show in Theorem II. 2 that type II balanced 101 truncation also preserves mean-square asymptotic stability. The 102 proof differs significantly from the one given for type I. Using 103 this result, we are able to give a more compact proof of the error 104 bound, Theorem II.4, which exploits the stochastic bounded 105 real lemma [17].

106 We illustrate our results by analytical and numerical exam107 ples in Section IV.

\section{II. TYPE I BALANCED TRUNCATION}

109 Consider a stochastic linear control system of Itô-type

$$
d x=A x d t+\sum_{j=1}^{k} N_{j} x d w_{j}+B u d t, \quad y=C x
$$

where $w_{j}=\left(w_{j}(t)\right)_{t \in \mathbb{R}_{+}}$are uncorrelated zero-mean real 110 Wiener processes on a probability space $(\Omega, \mathcal{F}, \mu)$ with respect 111 to an increasing family $\left(\mathcal{F}_{t}\right)_{t \in \mathbb{R}_{+}}$of $\sigma$-algebras $\mathcal{F}_{t} \subset \mathcal{F}$ (e.g., 112 [25], [26]).

To simplify the notation, we only consider the case $k=1114$ and set $w=w_{1}, N=N_{1}$. But all results can immediately be 115 generalized for $k>1$.

116

Let $L_{w}^{2}\left(\mathbb{R}_{+}, \mathbb{R}^{q}\right)$ denote the corresponding space of nonan- 117 ticipating stochastic processes $v$ with values in $\mathbb{R}^{q}$ and norm 118

$$
\|v(\cdot)\|_{L_{w}^{2}}^{2}:=\mathcal{E}\left(\int_{0}^{\infty}\|v(t)\|^{2} d t\right)<\infty
$$

where $\mathcal{E}$ denotes expectation.

Let the homogeneous equation $d x=A x d t+N x d w$ be 120 asymptotically mean-square-stable, i.e., $\mathcal{E}\left(\|x(t)\|^{2}\right) \stackrel{t \rightarrow \infty}{\longrightarrow} 0$, for 121 all solutions $x$.

Then, by Theorem A.1, the equations

$$
\begin{aligned}
& A^{T} Q+Q A+N^{T} Q N=-C^{T} C \\
& A P+P A^{T}+N P N^{T}=-B B^{T}
\end{aligned}
$$

have unique solutions $Q \geq 0$ and $P \geq 0$. If the system is 124 observable and reachable (see Theorem A.8), then $Q$ and $P$ are 125 nonsingular, and thus positive definite.

A similarity transformation

$$
(A, N, B, C) \mapsto\left(S^{-1} A S, S^{-1} N S, S^{-1} B, C S\right)
$$

of the system implies the contragradient transformation as

$$
(Q, P) \mapsto\left(S^{T} Q S, S^{-1} P S^{-T}\right) .
$$

Choosing, e.g., $S=L V \Sigma^{-1 / 2}$, with Cholesky factorizations 129 $L L^{T}=P, R^{T} R=Q$ and a singular value decomposition 130 $R L=U \Sigma V^{T}$, we obtain $S^{-1}=\Sigma^{-1 / 2} U^{T} R$ and

$$
S^{T} Q S=S^{-1} P S^{-T}=\Sigma=\operatorname{diag}\left(\sigma_{1}, \ldots, \sigma_{n}\right) .
$$

After suitable partitioning

$$
\Sigma=\left[\begin{array}{cc}
\Sigma_{1} & 0 \\
0 & \Sigma_{2}
\end{array}\right] \quad S=\left[\begin{array}{ll}
S_{1} & S_{2}
\end{array}\right] \quad S^{-1}=\left[\begin{array}{l}
T_{1} \\
T_{2}
\end{array}\right]
$$

a truncated system is given in the form

$$
\left(A_{11}, N_{11}, B_{1}, C_{1}\right)=\left(T_{1} A S_{1}, T_{1} N S_{1}, T_{1} B, C S_{1}\right) .
$$

The following result has been proven in [24].

Theorem I.1: Let $A, N \in \mathbb{R}^{n \times n}$ satisfy

$$
\sigma(I \otimes A+A \otimes I+N \otimes N) \subset \mathbb{C}_{-} .
$$

For a block-diagonal matrix $\Sigma=\operatorname{diag}\left(\Sigma_{1}, \Sigma_{2}\right)>0$ with 136 $\sigma\left(\Sigma_{1}\right) \cap \sigma\left(\Sigma_{2}\right)=\emptyset$, assume that

$$
A^{T} \Sigma+\Sigma A+N^{T} \Sigma N \leq 0 \text { and } A \Sigma+\Sigma A^{T}+N \Sigma N^{T} \leq 0 .
$$

Then, with the usual partitioning of $A$ and $N$, we have 
139 Its implication for mean-square stability of the truncated system 140 is immediate.

141 Corollary I.2: Consider an asymptotically mean square sta142 ble stochastic linear system

$$
d x=A x d t+N x d w .
$$

143 Assume that a matrix $\Sigma=\operatorname{diag}\left(\Sigma_{1}, \Sigma_{2}\right)$ is given as in 144 Theorem I.1 and $A$ and $N$ are partitioned accordingly. Then the 145 truncated system

$$
d x_{r}=A_{11} x_{r} d t+N_{11} x_{r} d w
$$

146 is also asymptotically mean square stable.

147 If the diagonal entries of $\Sigma_{2}$ are small, it is expected that the 148 truncation error is small. In fact this is supported by an $H^{2}$-error 149 bound obtained in [20]. Additionally, however, from the de150 terministic situation (see [2], [6]), one would also hope for an $151 H^{\infty}$-type error bound of the form

$$
\left\|y-y_{r}\right\|_{L_{w}^{2}\left(\mathbb{R}_{+}, \mathbb{R}^{p}\right)} \stackrel{?}{\leq} \alpha\left(\operatorname{trace} \Sigma_{2}\right)\|u\|_{L_{w}^{2}\left(\mathbb{R}_{+}, \mathbb{R}^{m}\right)}
$$

152 with some real number $\alpha>0$. The following example shows 153 that no such general $\alpha$ exists.

154 Example I.3: Let $A=-\left[\begin{array}{cc}1 & 0 \\ 0 & a^{2}\end{array}\right]$ with $a>1, \quad N=$ $155\left[\begin{array}{ll}0 & 0 \\ 1 & 0\end{array}\right], B=\left[\begin{array}{l}1 \\ 0\end{array}\right], C=\left[\begin{array}{ll}0 & 1\end{array}\right]$.

156 Solving (6) with equality, we get $P=\left[\begin{array}{cc}\frac{1}{2} & 0 \\ 0 & \frac{1}{4 a^{2}}\end{array}\right], Q=$ $157\left[\begin{array}{cc}\frac{1}{4 a^{2}} & 0 \\ 0 & \frac{1}{2 a^{2}}\end{array}\right]$ with $\sigma(P Q)=\left\{1 / 8 a^{2}, 1 / 8 a^{4}\right\}$ so that $\Sigma=$ $158 \operatorname{diag}\left(\sigma_{1}, \sigma_{2}\right)$, where $\sigma_{1}=1 / \sqrt{8} a$ and $\sigma_{2}=1 / \sqrt{8} a^{2}$. The sys159 tem is balanced by the transformation $S=\left[\begin{array}{cc}2 a^{2} & 0 \\ 0 & 1 / 2\end{array}\right]^{1 / 4}$. 160 Then $C S=\left(1 / 2^{1 / 4}\right)\left[\begin{array}{ll}0 & 1\end{array}\right]$ so that $C_{r}=0$ for the trun161 cated system of order 1 . Thus, the output of the reduced system 162 is $y_{r} \equiv 0$, and the truncation error $\left\|\mathbb{L}-\mathbb{L}_{r}\right\|$ is equal to the 163 stochastic $H^{\infty}$-norm (see [17]) of the original system

$$
\|\mathbb{L}\|=\sup _{x(0)=0,\|u\|_{L_{w}^{2}=1}}\|y\|_{L_{w}^{2}}
$$

164 We show now that this norm is equal to $1 / \sqrt{2} a=2 a \sigma_{2}$. 165 Thus, depending on $a$, the ratio of the truncation error and 166 trace $\Sigma_{2}=\sigma_{2}$ can be arbitrarily large.

167 According to the stochastic bounded real lemma, 168 Theorem A.5, $\|\mathbb{L}\|$ is the infimum over all $\gamma$ so that the Riccati 169 inequality

$$
\begin{aligned}
0 & <A^{T} X+X A+N^{T} X N-C^{T} C-\frac{1}{\gamma^{2}} X B B^{T} X \\
& =\left[\begin{array}{cc}
-2 x_{1}+x_{3}-\frac{1}{\gamma^{2}} x_{1}^{2} & -\left(a^{2}+1\right) x_{2}-\frac{1}{\gamma^{2}} x_{1} x_{2} \\
-\left(a^{2}+1\right) x_{2}-\frac{1}{\gamma^{2}} x_{1} x_{2} & -2 a^{2} x_{3}-\frac{1}{\gamma^{2}} x_{2}^{2}-1
\end{array}\right]
\end{aligned}
$$

170 possesses a solution $X=\left[\begin{array}{ll}x_{1} & x_{2} \\ x_{2} & x_{3}\end{array}\right]<0$.
If a given matrix $X$ satisfies this condition, then so does the 171 same matrix with $x_{2}$ replaced by 0 . Hence we can assume that 172 $x_{2}=0$, and end up with the two conditions $x_{3}<-\left(1 / 2 a^{2}\right) 173$ and (after multiplying the upper left entry with $-\gamma^{2}$ )

$$
\begin{aligned}
0 & >x_{1}^{2}+2 \gamma^{2} x_{1}-\gamma^{2} x_{3}=\left(x_{1}+\gamma^{2}\right)^{2}-\gamma^{2}\left(\gamma^{2}+x_{3}\right) \\
& >\left(x_{1}+\gamma^{2}\right)^{2}-\gamma^{2}\left(\gamma^{2}-\frac{1}{2 a^{2}}\right) .
\end{aligned}
$$

Thus necessarily $\gamma^{2}>1 / 2 a^{2}$, i.e., $\gamma>1 / \sqrt{2} a$. This already 175 proves that $\|\mathbb{L}\| \geq 1 / \sqrt{2} a=2 a \sigma_{2}$, which suffices to disprove 176 the existence of a general bound $\alpha$ in (9). Taking infima, it is 177 AQ1 easy to show that indeed $\|\mathbb{L}\|=1 / \sqrt{2} a$.

\section{TyPE II BALANCED TRUNCATION}

We now consider the inequalities (7).

Lemma II.1: Assume that $d x=A x d t+N x d w$ is asymptot- 181 ically mean-square-stable. Then inequality (7b) is solvable with 182 $P>0$.

Proof: By Theorem A.1, for a given $Y<0$, there exists a 184 $\tilde{P}>0$, so that $A^{T} \tilde{P}^{-1}+\tilde{P}^{-1} A+N^{T} \tilde{P}^{-1} N=Y$. Then $P=185$ $\varepsilon^{-1} \tilde{P}$, for sufficiently small $\varepsilon>0$, satisfies 186

$$
A^{T} P^{-1}+P^{-1} A+N^{T} P^{-1} N=\varepsilon Y<-\varepsilon^{2} \tilde{P}^{-1} B B^{T} \tilde{P}^{-1}
$$

so that $(7 b)$ holds even in the strict form.

It is easy to see that like in the previous section a state space 188 transformation

$$
(A, N, B, C) \mapsto\left(S^{-1} A S, S^{-1} N S, S^{-1} B, C S\right)
$$

leads to a contragradient transformation $Q \mapsto S^{T} Q S, P \mapsto 190$ $S^{-1} P S^{-T}$ of the solutions. That is, $Q$ and $P$ satisfy (7a) 191 and (7b), if and only if $S^{T} Q S$ and $S^{-1} P S^{-T}$ do so for the 192 transformed data. As before, we can assume the system to be 193 balanced with

$$
Q=P=\Sigma=\operatorname{diag}\left(\sigma_{1} I, \ldots, \sigma_{\nu} I\right)=\left[\begin{array}{ll}
\Sigma_{1} & \\
& \Sigma_{2}
\end{array}\right]
$$

where $\sigma_{1}>\cdots>\sigma_{\nu}>0$ and $\sigma\left(\Sigma_{1}\right)=\left\{\sigma_{1}, \ldots, \sigma_{r}\right\}, \sigma\left(\Sigma_{2}\right)=195$ $\left\{\sigma_{r+1}, \ldots, \sigma_{\nu}\right\}$. Hence, we will now assume (after balancing) 196 that a diagonal matrix $\Sigma$ as in (11) is given which satisfies

$$
\begin{gathered}
A^{T} \Sigma+\Sigma A+N^{T} \Sigma N \leq-C^{T} C \\
A^{T} \Sigma^{-1}+\Sigma^{-1} A+N^{T} \Sigma^{-1} N \leq-\Sigma^{-1} B B^{T} \Sigma^{-1} .
\end{gathered}
$$

Partitioning $A, N, B, C$ like $\Sigma$, we write the system as

$$
\begin{aligned}
d x_{1} & =\left(A_{11} x_{1}+A_{12} x_{2}+B_{1} u\right) d t+\left(N_{11} x_{1}+N_{12} x_{2}\right) d w \\
d x_{2} & =\left(A_{21} x_{1}+A_{22} x_{2}+B_{2} u\right) d t+\left(N_{21} x_{1}+N_{22} x_{2}\right) d w \\
y & =C_{1} x_{1}+C_{2} x_{2} .
\end{aligned}
$$

The reduced system obtained by truncation is

$$
d x_{r}=\left(A_{11} x_{r}+B_{1} u\right) d t+N_{11} x_{r} d w \quad y_{r}=C_{1} x_{r}
$$


200 The index $r$ is the number of different singular values $\sigma_{j}$ that 201 have been kept in the reduced system. In the following subsec202 tions, we consider matrices:

$$
A=\left[\begin{array}{ll}
A_{11} & A_{12} \\
A_{21} & A_{22}
\end{array}\right] \quad N=\left[\begin{array}{ll}
N_{11} & N_{12} \\
N_{21} & N_{22}
\end{array}\right]
$$

$203 \Sigma=\operatorname{diag}\left(\Sigma_{1}, \Sigma_{2}\right)$ as in (11), and equations of the form

$$
\begin{aligned}
A^{T} \Sigma+\Sigma A+N^{T} \Sigma N & =-\tilde{C}^{T} \tilde{C} \\
A^{T} \Sigma^{-1}+\Sigma^{-1} A+N^{T} \Sigma^{-1} N & =-\tilde{B} \tilde{B}^{T}
\end{aligned}
$$

204 with arbitrary right-hand sides $-\tilde{C}^{T} \tilde{C} \leq 0$ and $-\tilde{B} \tilde{B}^{T} \leq 0$.

\section{A. Preservation of Asymptotic Stability}

206 The following theorem is the main new result of this paper.

207 Theorem II.2: Let $A$ and $N$ be given such that

$$
\sigma(I \otimes A+A \otimes I+N \otimes N) \subset \mathbb{C}_{-} .
$$

208 Assume further that for a block-diagonal matrix $\Sigma=$ $209 \operatorname{diag}\left(\Sigma_{1}, \Sigma_{2}\right)>0$ with $\sigma\left(\Sigma_{1}\right) \cap \sigma\left(\Sigma_{2}\right)=\emptyset$, we have

$$
\begin{array}{r}
A^{T} \Sigma+\Sigma A+N^{T} \Sigma N \leq 0 \\
A^{T} \Sigma^{-1}+\Sigma^{-1} A+N^{T} \Sigma^{-1} N \leq 0 .
\end{array}
$$

210 Then, with the usual partitioning of $A$ and $N$, we have

$$
\sigma\left(I \otimes A_{11}+A_{11} \otimes I+N_{11} \otimes N_{11}\right) \subset \mathbb{C}_{-} .
$$

211 Again we have an immediate interpretation in terms of mean212 square stability of the truncated system.

213 Corollary II.3: Consider an asymptotically mean square 214 stable stochastic linear system

$$
d x=A x d t+N x d w .
$$

215 Assume that a matrix $\Sigma=\operatorname{diag}\left(\Sigma_{1}, \Sigma_{2}\right)$ is given as in 216 Theorem II.2 and $A$ and $N$ are partitioned accordingly. Then 217 the truncated system

$$
d x_{r}=A_{11} x_{r} d t+N_{11} x_{r} d w
$$

218 is also asymptotically mean square stable.

219 Proof of Theorem II.2: Note that the inequalities (15) are 220 equivalent to the equations (13) with appropriate right-hand 221 sides $-\tilde{C}^{T} \tilde{C}$ and $-\tilde{B} \tilde{B}^{T}$. In accordance with the partitioning 222 of $A, N$, and $\Sigma$, each matrix equation (13a) and (13b) consists 223 of three blocks.

224 By way of contradiction, we assume that (16) does not hold. 225 Then by Theorem A.3, there exist $V \geq 0, V \neq 0, \alpha \geq 0$ such that

$$
A_{11} V+V A_{11}^{T}+N_{11} V N_{11}^{T}=\alpha V .
$$

226 Taking the scalar product of the left upper block of (13a) with $227 V$, we obtain $0 \geq \alpha \operatorname{trace}\left(\Sigma_{1} V\right)$ whence $\alpha=0$ and $\tilde{C}_{1} V=0$, $228 N_{21} V=0$ by Corollary A.4. Hence

$$
\left(A_{11}^{T} \Sigma_{1}+\Sigma_{1} A_{11}+N_{11}^{T} \Sigma_{1} N_{11}\right) V=0 .
$$

229 Analogously, we have $\tilde{B}_{1}^{T} V=0$.
In particular, from $N_{21} V=0$, we get

$$
\left(\mathcal{L}_{A}^{*}+\Pi_{N}^{*}\right)\left(\left[\begin{array}{ll}
V & 0 \\
0 & 0
\end{array}\right]\right)=\left[\begin{array}{cc}
0 & V A_{21}^{T} \\
A_{21} V & 0
\end{array}\right] .
$$

We will show that $A_{21} V=0$, which implies

$$
0 \in \sigma(I \otimes A+A \otimes I+N \otimes N)
$$

in contradiction to (14), and thus finishes the proof.

We first show that $\operatorname{Im} V$ is invariant under $A_{11}$ and $N_{11}$. To 233 this end, let $V z=0$. Then by (17)

$$
0=z^{T}\left(A_{11} V+V A_{11}^{T}+N_{11} V N_{11}^{T}\right) z=z^{T} N_{11} V N_{11}^{T} z
$$

whence also $V N_{11}^{T} z=0$, i.e., $N_{11}^{T} z \in \operatorname{Ker} V$. From this, we have 235

$$
0=\left(A_{11} V+V A_{11}^{T}+N_{11} V N_{11}^{T}\right) z=V A_{11}^{T} z
$$

implying $A_{11}^{T} z \in \operatorname{Ker} V$. Thus, $A_{11}^{T} \operatorname{Ker} V \subset \operatorname{Ker} V$ and 236 $N_{11}^{T} \operatorname{Ker} V \subset \operatorname{Ker} V$.

237

Since $\operatorname{Ker} V=(\operatorname{Im} V)^{\top}$, it follows further that $\operatorname{Im} V$ is invari- 238 ant under $A_{11}$ and $N_{11}$.

239

Let $V=V_{1} V_{1}^{T}$, where $V_{1}$ has full column rank, i.e., 240 $\operatorname{det} V_{1}^{T} V_{1} \neq 0$. Then by the invariance, there exist square 241 matrices $X$ and $Y$, such that

$$
A_{11} V_{1}=V_{1} X \quad N_{11} V_{1}=V_{1} Y .
$$

It follows that

$$
\begin{aligned}
0 & =A_{11} V_{1} V_{1}^{T}+V_{1} V_{1}^{T} A_{11}^{T}+N_{11} V_{1} V_{1}^{T} N_{11}^{T} \\
& =V_{1}\left(X+X^{T}+Y Y^{T}\right) V_{1}^{T}
\end{aligned}
$$

whence $X+X^{T}+Y Y^{T}=0$. Moreover, from (18), we get 244

$$
\begin{aligned}
A_{11}^{T} \Sigma_{1} V_{1} & =-\Sigma_{1} A_{11} V_{1}-N_{11}^{T} \Sigma_{1} N_{11} V_{1} \\
& =-\Sigma_{1} V_{1} X-N_{11}^{T} \Sigma_{1} V_{1} Y .
\end{aligned}
$$

Using this substitution in the following computation, we obtain 245

$$
\begin{aligned}
0 \geq & V_{1}^{T} \Sigma_{1}^{2}\left(A_{11}^{T} \Sigma_{1}^{-1}+\Sigma_{1}^{-1} A_{11}+N_{11}^{T} \Sigma_{1}^{-1} N_{11}\right) \Sigma_{1}^{2} V_{1} \\
= & -V_{1}^{T} \Sigma_{1}^{3} V_{1} X-X^{T} V_{1}^{T} \Sigma_{1}^{3} V_{1} \\
& -V_{1}^{T} \Sigma_{1}^{2} N_{11}^{T} \Sigma_{1} V_{1} Y-Y^{T} V_{1}^{T} \Sigma_{1} N_{11} \Sigma_{1}^{2} V_{1} \\
& +V_{1}^{T} \Sigma_{1}^{2} N_{11}^{T} \Sigma_{1}^{-1} N_{11} \Sigma_{1}^{2} V_{1} .
\end{aligned}
$$

Taking the trace in (21), we have

$$
0=\operatorname{trace}\left[\begin{array}{c}
V_{1} Y \\
V_{1}
\end{array}\right]^{T} M\left[\begin{array}{c}
V_{1} Y \\
V_{1}
\end{array}\right]
$$

where

$$
M=\left[\begin{array}{cc}
\Sigma_{1}^{3} & -\Sigma_{1} N_{11} \Sigma_{1}^{2} \\
-\Sigma_{1}^{2} N_{11}^{T} \Sigma_{1} & \Sigma_{1}^{2} N_{11}^{T} \Sigma_{1}^{-1} N_{11} \Sigma_{1}^{2}
\end{array}\right]
$$

is positive semidefinite

$$
\left[\begin{array}{cc}
\Sigma_{1}^{3} & -\Sigma_{1} N_{11} \Sigma_{1}^{2} \\
-\Sigma_{1}^{2} N_{11}^{T} \Sigma_{1} & \Sigma_{1}^{2} N_{11}^{T} \Sigma_{1}^{-1} N_{11} \Sigma_{1}^{2}
\end{array}\right]\left[\begin{array}{c}
V_{1} Y \\
V_{1}
\end{array}\right]=0
$$


249 The first block row then implies $N_{11} \Sigma_{1}^{2} V_{1}=\Sigma_{1}^{2} V_{1} Y$. From 250 (21), using also (20) again, we thus have

$$
\begin{aligned}
0 & =\left(A_{11}^{T} \Sigma_{1}^{-1}+\Sigma_{1}^{-1} A_{11}+N_{11}^{T} \Sigma_{1}^{-1} N_{11}\right) \Sigma_{1}^{2} V_{1} \\
& =-\Sigma_{1} V_{1} X-N_{11}^{T} \Sigma_{1} V_{1} Y+\Sigma_{1}^{-1} A_{11} \Sigma_{1}^{2} V_{1}+N_{11}^{T} \Sigma_{1} V_{1} Y \\
& =-\Sigma_{1} V_{1} X+\Sigma_{1}^{-1} A_{11} \Sigma_{1}^{2} V_{1}
\end{aligned}
$$

251 i.e., $A_{11} \Sigma_{1}^{2} V_{1}=\Sigma_{1}^{2} V_{1} X$. It follows that for arbitrary $k \in \mathbb{N}$, the 252 eigenvector $V$ in (17) can be replaced by

$$
\Sigma_{1}^{2 k} V \Sigma_{1}^{2 k}=\Sigma_{1}^{2 k} V_{1} V_{1}^{T} \Sigma_{1}^{2 k}
$$

253 because

$$
\begin{aligned}
0= & \Sigma_{1}^{2} V_{1}\left(X+X^{T}+Y Y^{T}\right) V_{1}^{T} \Sigma_{1}^{2} \\
= & A_{11}\left(\Sigma_{1}^{2} V_{1} V_{1}^{T} \Sigma_{1}^{2}\right)+\left(\Sigma_{1}^{2} V_{1} V_{1}^{T} \Sigma_{1}^{2}\right) A_{11}^{T} \\
& +N_{11}\left(\Sigma_{1}^{2} V_{1} V_{1}^{T} \Sigma_{1}^{2}\right) N_{11}^{T} .
\end{aligned}
$$

254 Induction leads to

$$
\begin{aligned}
0=A_{11}\left(\Sigma_{1}^{2 k} V_{1} V_{1}^{T} \Sigma_{1}^{2 k}\right)+\left(\Sigma_{1}^{2 k} V_{1} V_{1}^{T} \Sigma_{1}^{2 k}\right) A_{11}^{T} \\
+
\end{aligned}
$$

255 As above, we conclude that $N_{21} \Sigma_{1}^{2 k} V_{1}=0, \tilde{C}_{1} \Sigma_{1}^{2 k} V_{1}=0$, and $256 \tilde{B}_{1}^{T} \Sigma_{1}^{2 k} V_{1}=0$. Multiplying the lower left blocks of (13a) and 257 (13b) with $\Sigma_{1}^{2(k-1)} V_{1}$ and $\Sigma_{1}^{2 k} V_{1}$, respectively, we get

$$
\begin{aligned}
A_{12}^{T} \Sigma_{1}^{2 k-1} V_{1}+\Sigma_{2} A_{21} \Sigma_{1}^{2(k-1)} V_{1}+N_{12}^{T} \Sigma_{1}^{2 k-1} V_{1} Y & =0 \\
A_{12}^{T} \Sigma_{1}^{2 k-1} V_{1}+\Sigma_{2}^{-1} A_{21} \Sigma_{1}^{2 k} V_{1}+N_{12}^{T} \Sigma_{1}^{2 k-1} V_{1} Y & =0 .
\end{aligned}
$$

258 Hence (after multiplication with $\Sigma_{2}$ ), for all $k \geq 1$, we have

$$
\begin{aligned}
\Sigma_{2}^{2} A_{21} \Sigma_{1}^{2(k-1)} V_{1} & =-\Sigma_{2}\left(A_{12}^{T} \Sigma_{1}^{2 k-1} V_{1}+N_{12}^{T} \Sigma_{1}^{2 k-1} V_{1} Y\right) \\
& =A_{21} \Sigma_{1}^{2 k} V_{1} .
\end{aligned}
$$

259 Applying this identity repeatedly, we get

$$
A_{21} \Sigma_{1}^{2 k} V_{1}=\Sigma_{2}^{2 k} A_{21} V_{1} \quad \text { for all } \quad k \in \mathbb{N} .
$$

260 If $\mu$ is the minimal polynomial of $\Sigma_{1}^{2}$, then $\sigma\left(\Sigma_{1}\right) \cap \sigma\left(\Sigma_{2}\right)=\emptyset$ 261 implies $\operatorname{det} \mu\left(\Sigma_{2}^{2}\right) \neq 0$ and

$$
0=A_{21} \mu\left(\Sigma_{1}^{2}\right) V_{1}=\mu\left(\Sigma_{2}^{2}\right) A_{21} V_{1}
$$

262 whence $A_{21} V_{1}=0$ and also $A_{21} V=0$. Hence we obtain the 263 contradiction (19).

\section{B. Error Estimate}

265 The following theorem has been proven in [19] using LMI266 techniques. Exploiting the stability result in the previous sub267 section, we can give a slightly more compact proof based on 268 the stochastic bounded real lemma, Theorem A.6.

269 Theorem II.4: Let $A$ and $N$ satisfy
Assume furthermore that for $\Sigma=\operatorname{diag}\left(\Sigma_{1}, \Sigma_{2}\right)>0$ with $\Sigma_{2}=270$ $\operatorname{diag}\left(\sigma_{r+1} I, \ldots, \sigma_{\nu} I\right)$ and $\sigma\left(\Sigma_{1}\right) \cap \sigma\left(\Sigma_{2}\right)=\emptyset$, the following 271 Lyapunov inequalities hold:

$$
\begin{gathered}
A^{T} \Sigma+\Sigma A+N^{T} \Sigma N \leq-C^{T} C \\
A^{T} \Sigma^{-1}+\Sigma^{-1} A+N^{T} \Sigma^{-1} N \leq-\Sigma^{-1} B B^{T} \Sigma^{-1} .
\end{gathered}
$$

If $x(0)=0$ and $x_{r}(0)=0$, then for all $T>0$, it holds that

$$
\left\|y-y_{r}\right\|_{L_{w}^{2}([0, T])} \leq 2\left(\sigma_{r+1}+\cdots+\sigma_{\nu}\right)\|u\|_{L_{w}^{2}([0, T])} .
$$

Proof: We adapt a proof for deterministic systems, e.g., 274 [2, Th. 7.9]. In the central argument we treat the case where 275 $\Sigma_{2}=\sigma_{\nu} I$ and show that

$$
\left\|y-y_{\nu-1}\right\|_{L_{w}^{2}[0, T]} \leq 2 \sigma_{\nu}\|u\|_{L_{w}^{2}[0, T]} .
$$

From the left upper blocks of (13a) and (13b), we can see 277 that also

$$
\begin{aligned}
A_{11}^{T} \Sigma_{1}+\Sigma_{1} A_{11}+N_{11}^{T} \Sigma_{1} N_{11} & \leq-C_{1}^{T} C_{1} \\
A_{11}^{T} \Sigma_{1}^{-1}+\Sigma_{1}^{-1} A_{11}+N_{11}^{T} \Sigma_{1}^{-1} N_{11} & \leq-\Sigma_{1}^{-1} B_{1} B_{1}^{T} \Sigma_{1}^{-1} .
\end{aligned}
$$

Hence we can repeat the above argument to remove $\sigma_{\nu-1}, 279$ $\ldots, \sigma_{r+1}$ successively. By the triangle inequality we find that 280

$$
\begin{aligned}
\left\|y-y_{r}\right\|_{L_{w}^{2}[0, T]} & \leq \sum_{j=r}^{\nu-1}\left\|y_{j+1}-y_{j}\right\|_{L_{w}^{2}[0, T]} \\
& \leq 2\left(\sigma_{\nu}+\cdots+\sigma_{r+1}\right)\|u\|_{L_{w}^{2}[0, T]} .
\end{aligned}
$$

which then concludes the proof.

To prove (22), we make use of the stochastic bounded real 282 lemma. In the following let $r=\nu-1$ and consider the error 283 system defined by:

$$
\begin{aligned}
d x_{e} & =A_{e} x_{e} d t+N_{e} x_{e} d w+B_{e} u d t \\
y_{e} & =C_{e} x_{e}=y-y_{r}
\end{aligned}
$$

where

$$
\begin{aligned}
x_{e} & =\left[\begin{array}{l}
x_{1} \\
x_{2} \\
x_{r}
\end{array}\right] \quad A_{e}=\left[\begin{array}{ccc}
A_{11} & A_{12} & 0 \\
A_{21} & A_{22} & 0 \\
0 & 0 & A_{11}
\end{array}\right] \\
N_{e} & =\left[\begin{array}{ccc}
N_{11} & N_{12} & 0 \\
N_{21} & N_{22} & 0 \\
0 & 0 & N_{11}
\end{array}\right] \quad B_{e}=\left[\begin{array}{l}
B_{1} \\
B_{2} \\
B_{1}
\end{array}\right] \\
C_{e} & =\left[\begin{array}{lll}
C_{1} & C_{2} & -C_{1}
\end{array}\right] .
\end{aligned}
$$

Applying the state space transformation

$$
\sigma(I \otimes A+A \otimes I+N \otimes N) \subset \mathbb{C}_{-} .
$$

$$
\left[\begin{array}{c}
\tilde{x}_{1} \\
\tilde{x}_{2} \\
\tilde{x}_{r}
\end{array}\right]=\left[\begin{array}{c}
x_{1}-x_{r} \\
x_{2} \\
x_{1}+x_{r}
\end{array}\right]=\underbrace{\left[\begin{array}{ccc}
I_{r} & 0 & -I_{r} \\
0 & I_{n-r} & 0 \\
I_{r} & 0 & I_{r}
\end{array}\right]}_{=S^{-1}}\left[\begin{array}{l}
x_{1} \\
x_{2} \\
x_{r}
\end{array}\right]
$$


287 we obtain the transformed system

$$
\begin{aligned}
& \tilde{A}_{e}=S^{-1} A_{e} S=\left[\begin{array}{ccc}
A_{11} & A_{12} & 0 \\
\frac{1}{2} A_{21} & A_{22} & \frac{1}{2} A_{21} \\
0 & A_{12} & A_{11}
\end{array}\right] \\
& \tilde{N}_{e}=S^{-1} N_{e} S=\left[\begin{array}{ccc}
N_{11} & N_{12} & 0 \\
\frac{1}{2} N_{21} & N_{22} & \frac{1}{2} N_{21} \\
0 & N_{12} & N_{11}
\end{array}\right] \\
& \tilde{B}_{e}=S^{-1} B\left[\begin{array}{c}
0 \\
B_{2} \\
2 B_{1}
\end{array}\right] \\
& \tilde{C}_{e}=C_{e} S=\left[\begin{array}{lll}
C_{1} & C_{2} & 0
\end{array}\right] .
\end{aligned}
$$

288 By Theorem A.6, we have $\left\|\mathbb{L}_{e}\right\| \leq 2 \sigma_{\nu}$, if the Riccati inequality

$$
\begin{aligned}
\mathcal{R}_{\sigma_{\nu}}(X)=\tilde{A}_{e}^{T} X+X \tilde{A}_{e}+\tilde{N}_{e}^{T} X \tilde{N}_{e}+\tilde{C}_{e}^{T} \tilde{C}_{e} & \\
& +\frac{1}{4 \sigma_{\nu}^{2}} X \tilde{B}_{e} \tilde{B}_{e}^{T} X \leq 0
\end{aligned}
$$

289 possesses a solution $X \geq 0$. In fact, such a solution is given by 290 the block-diagonal matrix

$$
X=\operatorname{diag}\left(\Sigma_{1}, 2 \Sigma_{2}, \sigma_{\nu}^{2} \Sigma_{1}^{-1}\right)=\operatorname{diag}\left(\Sigma_{1}, 2 \sigma_{\nu} I, \sigma_{\nu}^{2} \Sigma_{1}^{-1}\right)>0 .
$$

291 To verify this, we set $J=\left[\begin{array}{ll}0 & I \\ I & 0\end{array}\right]$ and

$$
M=J\left(A^{T} \Sigma^{-1}+\Sigma^{-1} A+N^{T} \Sigma^{-1} N+\Sigma^{-1} B B^{T} \Sigma^{-1}\right) J
$$

292 where $M \leq 0$ by (13b). Considering all blocks of (13a) and 293 (13b), a straight-forward computation yields

$$
\begin{aligned}
& \mathcal{R}_{\sigma_{\nu}}(X)=\left[\begin{array}{c}
A^{T} \Sigma+\Sigma A+N^{T} \Sigma N+C^{T} C \\
0
\end{array}\right. \\
& -\frac{\sigma_{\nu}}{2}\left[\begin{array}{c}
N_{21}^{T} \\
0 \\
-N_{21}^{T}
\end{array}\right]\left[\begin{array}{c}
N_{21}^{T} \\
0 \\
-N_{21}^{T}
\end{array}\right]^{T}+\sigma_{\nu}^{2}\left[\begin{array}{cc}
0 & 0 \\
0 & M
\end{array}\right] \leq 0
\end{aligned}
$$

294 which is inequality (23).

295 Example II.5: Let the system $(A, N, B, C)$ and $Q$ be as in 296 Example I.3. The matrix

$$
P=\left[\begin{array}{cc}
1+\sqrt{1-p} & 0 \\
0 & p
\end{array}\right]^{-1}>0, \text { where } 0<p \leq 1
$$

297 satisfies inequality (7b). As in Example I.3, we have $\mathbb{L}_{r}=0$ 298 for the corresponding reduced system of order 1 , so that the 299 truncation error again is $1 / \sqrt{2} a$, independently of $p \in] 0,1]$.

300 On the other hand we have

$$
\sigma_{2}^{2}=\min \sigma(P Q)=\frac{1}{4 a^{2}(1+\sqrt{1-p})} \geq \frac{1}{8 a^{2}}
$$

301 with equality for $p \rightarrow 0$. Theorem II.4 thus gives the sharp error 302 bound $2 \sigma_{2}=1 / \sqrt{2} a$. Note, that there is no $P>0$ satisfying (7b). 303 The previous example illustrates the problem of optimizing 304 over all solutions of inequality (7b).

\section{NuMERICAL EXAMPLES}

To compare the reduction methods, we need to compute $Q, P 306$ from (6) or (7). Instead of the inequalities (6a), (6b), (7a) we can 307 consider the corresponding equations, for which quite efficient 308 algorithms have been developed recently, e.g., [27]-[30]. These 309 also allow for a low-rank approximation of the solutions. In 310 contrast we cannot replace (7b) by the corresponding equation, 311 because this may not be solvable (see Example II.5). Even 312 worse, we neither have any solvability or uniqueness criteria 313 nor reliable algorithms.

314

Therefore, in general, we have to work with the inequality 315 (7b), which is solvable according to Lemma II.1, but of course 316 not uniquely solvable.

317

In view of our application, we aim at a solution $P$ of $(7 \mathrm{~b}), 318$ so that (some of) the eigenvalues of $P Q$ are particularly small, 319 since they provide the error bound. Choosing a matrix $Y<0320$ and a very small $\varepsilon$ along the lines of the proof of Lemma II.1 321 can be contrary to this aim. Hence some optimization over all 322 solutions of $(7 \mathrm{~b})$ is required.

Note also that a matrix $P>0$ satisfies (7b), if and only if it 324 satisfies the linear matrix inequality (LMI)

$$
\left[\begin{array}{cc}
P A^{T}+A P+B B^{T} & P N^{T} \\
N P & -P
\end{array}\right] \leq 0 .
$$

Thus, LMI optimal solution techniques are applicable. How- 326 ever, their complexity will be prohibitive for large-scale prob- 327 lems. Therefore further research for alternative methods to 328 solve (7b) adequately is required.

329

By $\mathbb{L}$ and $\mathbb{L}_{r}$, we always denote the original and the $r$-th 330 order approximated system. The stochastic $H^{\infty}$-type norm 331 $\left\|\mathbb{L}-\mathbb{L}_{r}\right\|$ is computed by a binary search of the infimum of all 332 $\gamma$ such that the Riccati inequality (10) is solvable. The latter is 333 solved via a Newton iteration as in [18]. Finally, the Lyapunov 334 equations (2) are solved by preconditioned Krylov subspace 335 methods described in [27].

Unfortunately, for small $\gamma$, i.e., for small approximation 337 errors, this method of computing the error runs into numerical 338 problems, because (10) contains the term $\gamma^{-2}$. This apparently 339 leads to cancellation phenomena in the Newton iteration, if, 340 e.g., $\gamma<10^{-7}$. Therefore we mainly concentrate on cases 341 where the error is larger, i.e., we make $r$ sufficiently small. $\quad 342$

\section{A. Type II Can be Better Than Type I}

In many examples we observe that type II reduction gives a 344 valid error bound, but the approximation error still is better with 345 type I. This, however, is not always true, as the example

346

$$
\left(A, N, B, C^{T}\right)=\left(\left[\begin{array}{cc}
-1 & 1 \\
0 & -1
\end{array}\right],\left[\begin{array}{ll}
0 & 0 \\
1 & 0
\end{array}\right],\left[\begin{array}{l}
0 \\
3
\end{array}\right],\left[\begin{array}{l}
3 \\
0
\end{array}\right]\right)
$$

shows. It can easily be verified that the type I Lyapunov 347 equations (6) are solved by

$$
Q=\left[\begin{array}{ll}
6 & 3 \\
3 & 3
\end{array}\right] \quad P=\left[\begin{array}{ll}
3 & 3 \\
3 & 6
\end{array}\right]
$$

The type II inequalities (7) are, e.g., solved by

$$
Q=\left[\begin{array}{ll}
6 & 3 \\
3 & 3
\end{array}\right] \quad P=\left[\begin{array}{rr}
8 & 0 \\
0 & 12
\end{array}\right]
$$


TABLE I

ERROR BOUNDS AND APPROXIMATION ERRORS FOR BOTH TYPES

\begin{tabular}{l||c|c}
\hline & $\sigma_{2}$ & $\left\|\mathbb{L}-\mathbb{L}_{1}\right\|$ \\
\hline I & 2.4853 & 3.9647 \\
\hline II & 6.9282 & 3.5614 \\
\hline
\end{tabular}

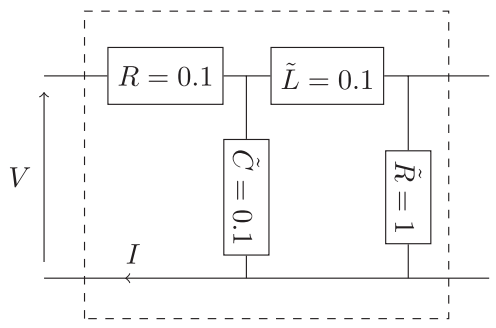

Fig. 1. Section of ladder network from [31].

350 If we reduce to order $r=1$, the type I approximation error is 351 larger than both the truncated singular value and the type II 352 approximation error; see Table I.

\section{B. Electrical Ladder Network With Perturbed Inductance}

354 As our first example with a physical background, we take 355 up the electrical ladder network described in [31], consisting of $356 n / 2$ sections with a capacitor $\tilde{C}$, inductor $\tilde{L}$ and two resistors $357 R$ and $\tilde{R}$ as depicted in Fig. 1 .

358 But following, e.g., [32], we assume that the inductance $\tilde{L}$ is 359 subject to stochastic perturbations. For simplicity, we replace the 360 inverse $\tilde{L}^{-1}$ formally by $L^{-1}+\dot{w}$ in all sections. Here $L=0.1$ 361 and $\dot{w}$ is white noise of a certain intensity $\sigma$, where we set $\sigma=1$, 362 e.g., for $n=6$, we have the system matrices

$$
\begin{aligned}
A & =\left[\begin{array}{cccccc}
\frac{-1}{\tilde{C} R} & \frac{-1}{\tilde{C}} & 0 & 0 & 0 & 0 \\
\frac{1}{L} & \frac{-R \tilde{R}}{L(R+R)} & \frac{-\tilde{R}}{L(R+R)} & 0 & 0 & 0 \\
0 & \frac{\tilde{R}}{\tilde{C}(R+\tilde{R})} & \frac{-1}{\tilde{C}(R+\tilde{R})} & \frac{-1}{C} & 0 & 0 \\
0 & 0 & \frac{1}{L} & \frac{-R \tilde{R}}{L(R+\tilde{R})} & \frac{-\tilde{R}}{L(R+\tilde{R})} & 0 \\
0 & 0 & 0 & \frac{\tilde{R}}{\tilde{C}(R+\bar{R})} & \frac{-1}{\tilde{C}(R+\tilde{R})} & \frac{-1}{\tilde{C}} \\
0 & 0 & 0 & 0 & \frac{1}{L} & \frac{-\tilde{R}}{L}
\end{array}\right] \\
N & =\left[\begin{array}{ccccccc}
0 & 0 & 0 & 0 & 0 & 0 \\
1 & \frac{-R \tilde{R}}{R+\tilde{R}} & \frac{-\tilde{R}}{R+\tilde{R}} & 0 & 0 & 0 \\
0 & 0 & 0 & 0 & 0 & 0 \\
0 & 0 & 1 & \frac{-R \tilde{R}}{R+\tilde{R}} & \frac{-\tilde{R}}{R+\tilde{R}} & 0 \\
0 & 0 & 0 & 0 & 0 & 0 \\
0 & 0 & 0 & 0 & 1 & -\tilde{R}
\end{array}\right] \\
B & =\left[\begin{array}{cccccccc}
\frac{1}{\tilde{C} R} & 0 & 0 & 0 & 0 & 0 & 0
\end{array}\right]^{T}
\end{aligned}
$$

363 For larger $n$, the band structure of $A$ and $N$ is extended 364 periodically. To see the behavior of our two methods, we reduce 365 from order $n=20$ to the orders $r=1,3,5, \ldots, 19$, and com366 pute both the theoretical bounds and the actual approximation 367 errors in the $H^{\infty}$-norm; see Fig. 2.

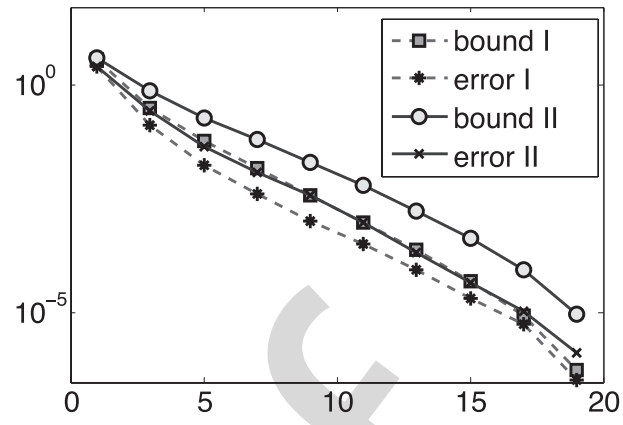

Fig. 2. In this example, for both types the bounds hold, and for all reduced orders, type I gives a smaller $H^{\infty}$-error than type II.
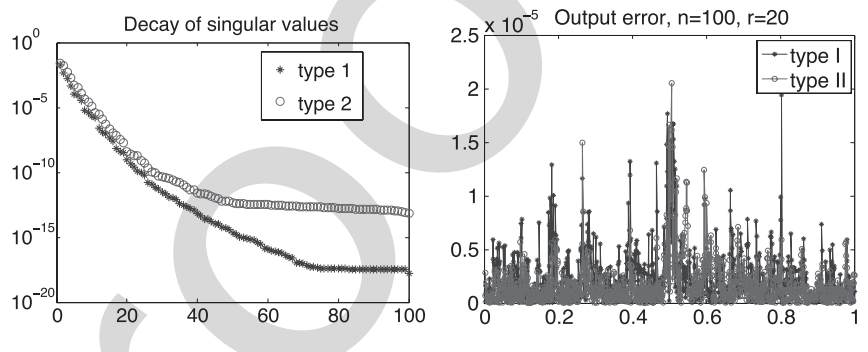

Fig. 3. Comparison of singular values and relative output error.

\section{Heat Transfer Problem}

As another example we consider a stochastic modification of 369 the heat transfer problem described in [14]. On the unit square 370 $\Omega=[0,1]^{2}$, the heat equation $x_{t}=\Delta x$ is given with Dirichlet 371 condition $x=u_{j}, j=1,2,3$, on three of the boundary edges 372 and a stochastic Robin condition $n \cdot \nabla x=(1 / 2+\dot{w}) x$ on the 373 fourth edge (where $\dot{w}$ stands for white noise). A standard five- 374 point finite-difference discretization on a $10 \times 10$ grid leads 375 to a modified Poisson matrix $A \in \mathbb{R}^{100 \times 100}$ and corresponding 376 matrices $N \in \mathbb{R}^{100 \times 100}$ and $B \in \mathbb{R}^{100 \times 3}$. We use the input 377

$$
u \equiv\left[\begin{array}{l}
1 \\
1 \\
1
\end{array}\right]
$$

and choose the average temperature as the output, i.e., $C=378$ $(1 / 100)[1, \ldots, 1]$. We apply balanced truncation of type I 379 and type II. For type II, an LMI-solver (MATLAB function 380 mincx) is used to compute $P$ as a solution of the LMI (24) 381 which minimizes trace $P$ or $\operatorname{trace} P Q$.

382

In the following figure (Fig. 3), we compare the reduced 383 systems of order $r=20$ for both types. The left diagram shows 384 the decay of the singular values. Since the LMI-solver was 385 called with tolerance level $10^{-9}$, only the first about 25 singular 386 values for type II have the correct order of magnitude. In this 387 region, the decay for both types is roughly linear. Some analysis 388 of this behavior for type I has been carried out in [28]. For 389 type II, so far no theoretical results are available.

390

The diagram on the right displays the approximation error 391 $\left\|y(t)-y_{r}(t)\right\|$ over a given time interval. For both types it has 392 the same order of magnitude. In fact, for many examples we 393 have observed both methods to yield very similar results. $\quad 394$

The estimated error norm $\sum_{j=r+1}^{n} \sigma_{j}$ and the actual approx- 395 imation error $\left\|\mathbb{L}-\mathbb{L}_{10}\right\|$ are given in Table II. 
TABLE II

ERROR BOUNDS AND APPROXIMATION ERRORS FOR BOTH TYPES

\begin{tabular}{l||c|c||c|c}
\hline & $\sum_{j=11}^{100} \sigma_{j}$ & $\left\|\mathbb{L}-\mathbb{L}_{10}\right\|$ & $\sum_{j=21}^{100} \sigma_{j}$ & $\left\|\mathbb{L}-\mathbb{L}_{20}\right\|$ \\
\hline I & $4.66 e-06$ & $9.30 e-06$ & $2.00 e-09$ & $9.65 e-09$ \\
\hline II & $1.75 e-05$ & $4.83 e-06$ & $1.72 e-08$ & $9.70 e-09$ \\
\hline
\end{tabular}

TABLE III

COMPARISON OF BOTH REDUCTION METHODS

\begin{tabular}{l||c|c}
\hline Type & I & II \\
\hline \hline Def. of $P, Q$ & $(6)$ & (7) \\
\hline Stability? & Yes, [24] & Yes, Thm. II.2 \\
\hline$H^{2}$-bound? & Yes, [20] & Yes, [33] \\
\hline$H^{\infty}$-bound? & No, Ex. I.3 & Yes, Thm. II.4 or [19] \\
\hline comput. cost & medium & high (via LMI) \\
\hline
\end{tabular}

397 As we can see, the upper error bound fails for type I, but is 398 correct for type II. Nevertheless, judging from the $H^{\infty}$ error, 399 neither of the types seems to be preferable over the other.

\section{D. Summary}

401 Clearly, higher dimensional examples are required to get 402 more insight. To this end, a more sophisticated method for the 403 solution of (24) is needed. With general-purpose LMI-software 404 on a standard Laptop, we hardly got higher than $n=100$.

405

406 Table III summarizes properties of our two methods.

407 As long as efficient algorithms for the solution of (7b) are not 408 available, practical evidence favors to use the type I method in 409 applications. Although there is no strict $H^{\infty}$-type error bound 410 for this case, in most examples the decay of singular values still 411 roughly indicates the decay of the approximation error.

\section{VI. CONCLUSIONS AND FUTURE WORK}

413 We have discussed two ways of generalizing balanced trun414 cation for stochastic linear systems. The main theoretical con415 tributions of this paper are the preservation of asymptotic 416 stability for type II balanced truncation proved in Theorem II.2 417 and the new proof of the $H^{\infty}$ error bound in Theorem II.4. 418 The efficient solution of the matrix inequality ( $7 b)$ is an open 419 issue and requires further research. The same is true for the 420 computation of the stochastic $H^{\infty}$-norm. Moreover, we are still 421 looking for adequate interpretations of our approaches, e.g., in 422 terms of energy minimization or Hankel operators. We hope to 423 trigger some research in this direction.

424

\section{APPENDIX A}

\section{Asymptotic Mean SQUare Stability}

Consider the stochastic linear system of Itô-type

$$
d x=A x d t+N x d w
$$

427 where $w=(w(t))_{t \in \mathbb{R}_{+}}$is a zero-mean real Wiener process on a 428 probability space $(\Omega, \mathcal{F}, \mu)$ with respect to an increasing family $429\left(\mathcal{F}_{t}\right)_{t \in \mathbb{R}_{+}}$of $\sigma$-algebras $\mathcal{F}_{t} \subset \mathcal{F}$ (e.g., [25], [26]).
Let $L_{w}^{2}\left(\mathbb{R}_{+}, \mathbb{R}^{q}\right)$ denote the corresponding space of nonan- 430 ticipating stochastic processes $v$ with values in $\mathbb{R}^{q}$ and norm $\quad 431$

$$
\|v(\cdot)\|_{L_{w}^{2}}^{2}:=\mathcal{E}\left(\int_{0}^{\infty}\|v(t)\|^{2} d t\right)<\infty
$$

where $\mathcal{E}$ denotes expectation. For initial data $x(0)=x_{0}$, the 432 solution can be written as $x(t)=\Phi(t) x_{0}$ with the fundamental 433 matrix solution $\Phi(t)$, satisfying $\Phi(0)=I$

434

By definition, system (25) is asymptotically mean-square- 435 stable, if $\mathcal{E}\left(\|x(t)\|^{2}\right) \stackrel{t \rightarrow \infty}{\longrightarrow} 0$, for all initial conditions $x_{0}$. In this 436 case, for simplicity, we also call the pair $(A, N)$ asymptotically 437 mean-square stable.

We have the following version of Lyapunov's matrix 439 theorem; see [23]. Here $\otimes$ denotes the Kronecker product. $\quad 440$

Theorem A.1: The following are equivalent.

(i) System (25) is asymptotically mean-square stable.

(ii) $\max \{\Re \lambda \| \lambda \in \sigma(A \otimes I+I \otimes A+N \otimes N)\}<0$ 442

(iii) $\exists Y>0: \exists X>0: A^{T} X+X A+N^{T} X N=-Y$

(iv) $\forall Y>0: \exists X>0$ : $A^{T} X+X A+N^{T} X N=-Y$

(v) $\forall Y \geq 0: \exists X \geq 0: A^{T} X+X A+N^{T} X N=-Y$

Remark A.2: The theorem (like all other results in this paper) 448 carries over to systems

$$
d x=A x d t+\sum_{j=1}^{k} N_{j} x d w_{j}
$$

with more than one noise term, and many more equivalent 450 criteria can be provided; see, e.g., [34] or [18, Th. 3.6.1]. 451

The following theorem does not require any stability assump- 452 tions (see [18, Th. 3.2.3]). It is central in the analysis of mean- 453 square stability.

Theorem A.3: Let

$$
\alpha=\max \{\Re \lambda \mid \lambda \in \sigma(A \otimes I+I \otimes A+N \otimes N)\} .
$$

Then there exists a nonnegative definite matrix $V \neq 0$, so that 456

$$
\left(\mathcal{L}_{A}^{*}+\Pi_{N}^{*}\right)(V)=A V+V A^{T}+N V N^{T}=\alpha V .
$$

We also note a simple consequence of this theorem [24, 457 Cor. 3.2]. Here $\langle Y, V\rangle=\operatorname{trace}(Y V)$ is the Frobenius inner 458 product for symmetric matrices.

Corollary A.4: Let $\alpha, V$ as in the theorem. For given $Y \geq 0460$ assume that

$$
\exists X>0: \mathcal{L}_{A}(X)+\Pi_{N}(X) \leq-Y .
$$

Then $\alpha \leq 0$. Moreover, if $\alpha=0$ then $Y V=V Y=0$.

\section{APPENDIX B}

\section{STOCHASTIC BOUNDED REAL LEMMA}

Now let us consider system (5) with input $u$ and output $y .465$ If $(A, N)$ is asymptotically mean-square stable, then (5) de- 466 fines an input output operator $\mathbb{L}: u \mapsto y$ from $L_{w}^{2}\left(\mathbb{R}, \mathbb{R}^{m}\right)$ to 467 $L_{w}^{2}\left(\mathbb{R}, \mathbb{R}^{p}\right)$, see [17]. By $\|\mathbb{L}\|$ we denote the induced operator 468 norm, which is an analogue of the deterministic $H^{\infty}$-norm. It 469 can be characterized by the stochastic bounded real lemma. $\quad 470$ 
Theorem A.5: [17] For $\gamma>0$, the following are equivalent.

(i) System (25) is asymptotically mean-square stable and $\|\mathbb{L}\|<\gamma$.

(ii) There exists a negative definite solution $X<0$ to the Riccati inequality

$$
A^{T} X+X A+N^{T} X N-C^{T} C-\gamma^{-2} X B B^{T} X>0 .
$$

(iii) There exists a positive definite solution $X>0$ to the Riccati inequality

$$
A^{T} X+X A+N^{T} X N+C^{T} C+\gamma^{-2} X B B^{T} X<0 .
$$

479 We have stated the obviously equivalent formulations (ii) and 480 (iii) to avoid confusion arising from different formulations 481 in the literature. Under additional assumptions also nonstrict 482 versions can be formulated. The following sufficient criterion 483 is given in [18, Cor. 2.2.3] (where also the signs are changed). 484 Unlike in the previous theorem, here asymptotic mean-square 485 stability is assumed at the outset.

486 Theorem A.6: Assume that (25) is asymptotically stable in 487 mean-square. If there exists a nonnegative definite matrix $X \geq 0$, 488 satisfying

$$
A^{T} X+X A+N^{T} X N+C^{T} C+\gamma^{-2} X B B^{T} X \leq 0
$$

489 then $\|\mathbb{L}\| \leq \gamma$.

490

491

492 Definition A.7: Consider system (5). A vector $v \in \mathbb{R}^{n}$ is 493 called unobservable, if the initial condition $x(0)=v$ with $u \equiv 0$ 494 produces the output $y \equiv 0$. The vector $v$ is called unreachable, 495 if $x(t) \neq v$ for all $t>0$ and any solution with initial value $496 x(0)=0$ and arbitrary input $u$.

497 If $(A, N)$ is asymptotically mean-square stable, then (see [14, 498 Th. 3.1]) the unobservable and the unreachable subspace can be 499 characterized as the kernels of $Q$ and $P$ defined by

$$
\begin{aligned}
& A^{T} Q+Q A+N^{T} Q N=-C^{T} C \\
& A P+P A^{T}+N P N^{T}=-B B^{T} .
\end{aligned}
$$

599 Theorem A.8: A state $v$ is

502 (a) unobservable, if and only if $Q v=0$.

503 (b) unreachable, if and only if $P v=0$.

504 In particular, the system is observable and reachable, if and only 505 if $Q>0$ and $P>0$.

\section{REFERENCES}

[6] B. C. Moore, "Principal component analysis in linear systems: controlla- 521 bility, observability, and model reduction," IEEE Trans. Autom. Control, 522 vol. AC-26, pp. 17-32, 1981.

[7] L. Pernebo and L. M. Silverman, "Model reduction via balanced state 524 space representations," IEEE Trans. Autom. Control, vol. AC-27, no. 2, 525 pp. 382-387, 1982.

[8] T. Stykel, "Analysis and numerical solution of generalized Lyapunov 527 equations," Ph.D. dissertation, Tech. Univ. Berlin, Berlin, Germany 2002. 528

[9] A. Shokoohi, L. M. Silverman, and P. M. Van Dooren, "Linear time- 529 variable systems: balancing and model reduction," IEEE Trans. Autom. 530 Control, vol. AC-28, no. 8, pp. 810-822, 1983.

[10] E. Verriest and T. Kailath, "On generalized balanced realizations," IEEE 532 Trans. Autom. Control, vol. AC-28, no. 8, pp. 833-844, 1983.

[11] H. Sandberg and A. Rantzer, "Balanced truncation of linear time-varying 534 systems," IEEE Trans. Autom. Control, vol. 49, no. 2, pp. 217-229, 535 Feb. 2004.

[12] S. A. Al-Baiyat, M. Bettayeb, and U. M. Al-Saggaf, "New model reduc- 537 tion scheme for bilinear systems," Int. J. Syst. Sci., vol. 25, pp. 1631-1642, 538 1994.

[13] W. S. Gray and J. Mesko, "Energy functions and algebraic Gramians 540 for bilinear systems," in Proc. 4th IFAC Nonlinear Control Syst. Design 541 Symp., Enschede, The Netherlands, 1998, pp. 103-108.

[14] P. Benner and T. Damm, "Lyapunov equations, energy functionals, and 543 model order reduction of bilinear and stochastic systems," SIAM J. 544 Control Optim., vol. 49, no. 2, pp. 686-711, 2011.

[15] J. M. A. Scherpen, "Balancing for nonlinear systems," Syst. Control Lett., 546 vol. 21, no. 2, pp. 143-153, 1993.

[16] W. M. Wonham, "Random differential equations in control theory," in 548 Probabilstic Methods in Applied Mathematics, A. T. Bharucha-Reid, Ed., 549 vol. 2. New York, NY, USA: Academic, 1970, pp. 131-212. 550

[17] D. Hinrichsen and A. J. Pritchard, "Stochastic $H_{\infty}$, , SIAM J. Control 551 Optim., vol. 36, no. 5, pp. 1504-1538, 1998.

[18] T. Damm, Rational Matrix Equations in Stochastic Control, ser. Lec- 553 ture Notes in Control and Information Sciences. New York, NY, USA: 554 Springer, 2004, no. 297.

[19] T. Damm and P. Benner, "Balanced truncation for stochastic linear sys- 556 tems with guaranteed error bound," in Proc. MTNS-2014, Groningen, 557 The Netherlands, 2014, pp. 1492-1497.

[20] P. Benner and M. Redmann, "Model reduction for stochastic systems," 559 Stochastic Partial Diff. Eq.: Anal. Comput., vol. 3, no. 3, pp. 291-338, 560 2015.

[21] F. R. Gantmacher, The Theory of Matrices, vol. 2. New York, NY, USA: 562 Chelsea, 1959.

[22] D. L. Kleinman, "On the stability of linear stochastic systems," IEEE 564 Trans. Autom. Control, vol. AC-14, pp. 429-430, 1969.

[23] R. Z. Khasminskij, Stochastic Stability of Differential Equations. 566 Alphen aan den Rijn, NL: Sijthoff \& Noordhoff, 1980.

[24] P. Benner, T. Damm, M. Redmann, and Y. Rocio Rodriguez Cruz, "Pos- 568 itive operators and stable truncation," Linear Algebra Appl., vol. 498, 569 Jun. 2016, pp. 74-87.

[25] L. Arnold, Stochastic Differential Equations: Theory and Applications. 571 Translation. New York, NY, USA: Wiley, 1974. 572

[26] B. Oeksendal, Stochastic Differential Equations, 5th ed. Berlin, 573 Germany: Springer-Verlag, 1998.

[27] T. Damm, "Direct methods and ADI-preconditioned Krylov subspace 575 methods for generalized Lyapunov equations," Numer. Linear Alg. Appl., 576 vol. 15 , no. 9 , pp. 853-871, 2008.

[28] P. Benner and T. Breiten, "Low rank methods for a class of generalized 578 Lyapunov equations and related issues," Numer. Math., vol. 124, no. 3, 579 pp. 441-470, 2013.

[29] D. Kressner and P. Sirković, "Greedy low-rank methods for solving 581 general linear matrix equations," Numer. Alg. and High-Performance 582 Comput. (ANCHP), Math. Inst. Comput. Sci. Eng. (MATHICSE), École 583 Polytechnique Fédérale de Lausanne, Lausanne, Switzerland, 2014. 584

[30] S. Shank, V. Simoncini, and D. Szyld, "Efficient low-rank solu- 585 tions of generalized Lyapunov equations," Dept. Math., Temple Univ., 586 Philadelphia, PA, USA, 2014.

[31] S. Gugercin and A. Antoulas, "A survey of model reduction by bal- 588 anced truncation and some new results," Int. J. Control, vol. 77, no. 8, 589 pp. 748-766, 2004.

[32] V. A. Ugrinovskii and I. R. Petersen, "Absolute stabilization and minimax 591 optimal control of uncertain systems with stochastic uncertainty," SIAM 592 J. Control Optim., vol. 37, no. 4, pp. 1089-1122, 1999.

[33] M. Redmann and P. Benner, "An $\mathcal{H}_{2}$-type error bound for balancing- 594 related model order reduction of linear systems with Lévy noise," Max 595 Planck Inst., Magdeburg, Germany, 2015. 596

[34] H. Schneider, "Positive operators and an inertia theorem," Numer. Math., 597 vol. 7, pp. 11-17, 1965. 


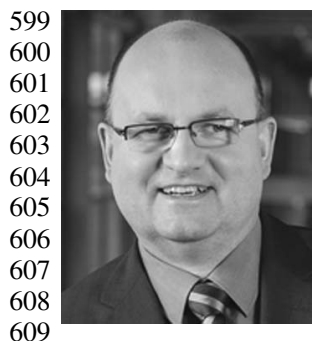

Peter Benner received the Diplom in mathematics from the RWTH Aachen, Aachen, Germany, in 1993. From 1993 to 1997, he worked on his Ph.D. at the University of Kansas, Lawrence, KS USA, and the TU Chemnitz-Zwickau, Germany, where he received the Ph.D. degree in February 1997. In 2001, he received the Habilitation in Mathematics from the University of Bremen, Bremen, Germany, where he held an Assistant Professor position from 1997 to 2001 .

After spending a term as a Visiting Associate 610 Professor at TU Hamburg-Harburg, Germany, he was a Lecturer in mathematics 611 at TU Berlin 2001-203. Since 2003, he has been a Professor for mathematics 612 in industry and technology at TU Chemnitz. In 2010, he was appointed as 613 one of the four directors of the Max Planck Institute for Dynamics of Com614 plex Technical Systems, Magdeburg, Germany. Since 2011, he has also been 615 an Honorary Professor at the Otto-von-Guericke University of Magdeburg. $616 \mathrm{He}$ was named Distinguished Professor at Shanghai University in 2015. His 617 research interests are in the areas of scientific computing, numerical mathemat618 ics, systems theory, and optimal control. A particular emphasis has been on 619 applying methods from numerical linear algebra and matrix theory in systems 620 and control theory. Recent research focuses on numerical methods for optimal 621 control of systems modeled by evolution equations (PDEs, DAEs, SPDEs), 622 model order reduction, preconditioning in optimal control and UQ problems, 623 and Krylov subspace methods for structured or quadratic eigenproblems. 624 Research in all these areas is accompanied by the development of algo625 rithms and mathematical software suitable for modern and high-performance 626 computer architectures.

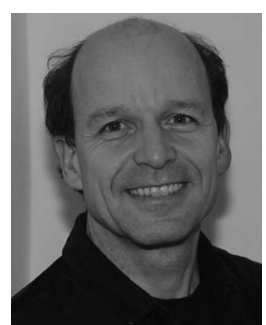

Tobias Damm received the diploma in mathemat- 627 ics from the University of Würzburg, Würzburg, 628 Germany, in 1996 and the doctoral degree from the 629 University of Bremen, Bremen, Germany, in 2002. 630

From 2002 to 2006 he held postdoctoral positions 631 at TU München and TU Braunschweig. He was 632 appointed Junior Professor at the TU Kaiserslautern 633 in 2006 and Associate Professor at the University 634 of Bayreuth in 2011. Since 2012 he has been a 635 Professor for systems and control theory at the TU 636 Kaiserslautern, Kaiserslautern, Germany. His re- 637 search interests encompass stochastic control systems, matrix equations, and 638 numerical methods.

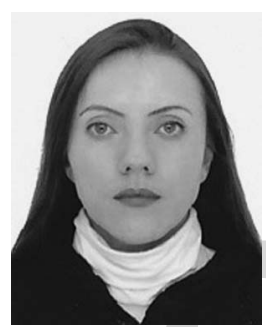

Yolanda Rocio Rodriguez Cruz received the B.Sc. 640 degree in physics from the National University of 641 Colombia, Bogotá, Colombia, in 2004, and the 642 M.Sc. degree in industrial mathematics from the TU 643 Kaiserslautern, Kaiserslautern, Germany, in 2008. 644

From 2009 to 2010, she was a Lecturer at the 645 District University Francisco José de Caldas, Bogotá, 646 Colombia. In the intersemestral period in 2011, she 647 was Lecturer at the National University of Colombia. 648 From 2011 to 2013, she was a Lecturer at the Univer- 649 sity of Bogotá Jorge Tadeo Lozano, Bogotá, Colombia. 650 She is currently a Ph.D. researcher at the TU Kaiserslautern. Her research has 651 been concerned with model reduction for stochastic and bilinear systems.

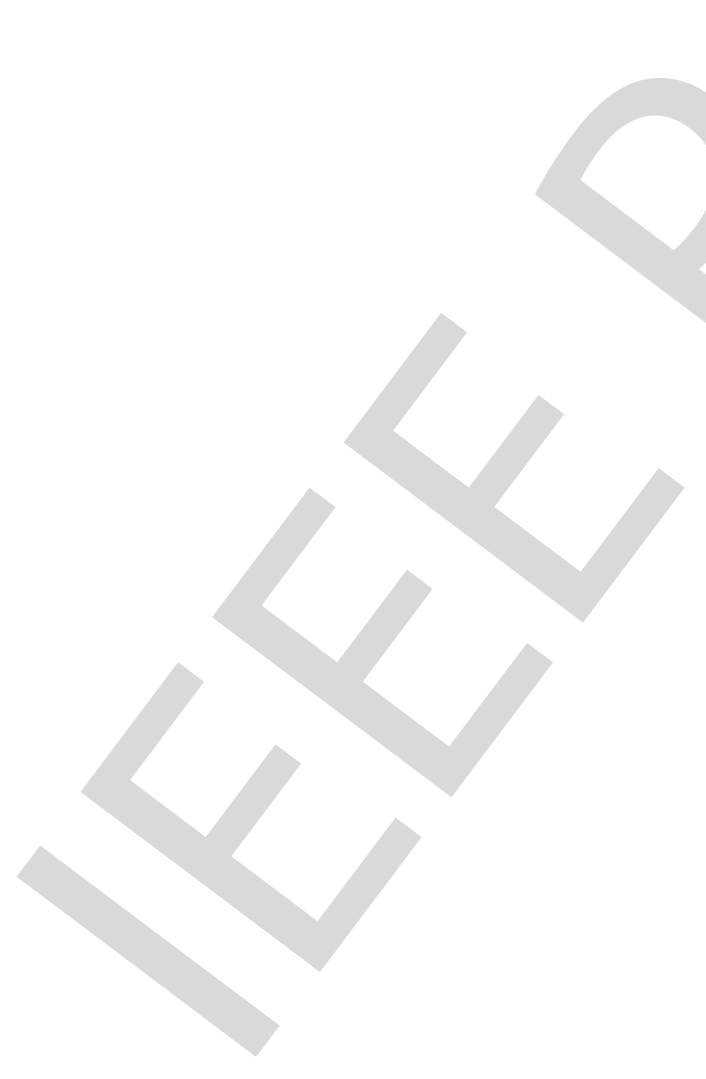




\section{AUTHOR QUERIES}

\section{AUTHOR PLEASE ANSWER ALL QUERIES}

\section{AQ1 = "infima" ok?}

AQ2 = Please provide department name in Ref. [8].

END OF ALL QUERIES 